\title{
Targeting macrophage polarization for therapy of diabesity-the feasibility of early improvement of insulin sensitivity and insulin resistance-a comprehensive systematic review
}

\begin{abstract}
The incidence of obesity is increasing in mammoth proportions so much so that associated comorbidity T2DM incidence is increasing markedly that we had to coin a term "Diabesity" to target both together. Earlier trying to review etiopathogenesis of both obesity and type $2 \mathrm{DM}$ we found that whatever drugs that are formulated usually do not work with some complications and till now other than bariatric surgery we have no permanent cure for long term maintenance. Further T2DM might be a disease of 2 etiopathogenesis, namely inflammatory, as well as metabolic. Although metabolic inflammation has the properties of being systemic, their common initiating point is tissue resident macrophages, whose successful physiological or abnormal pathological adaptation to its microenvironment dictates disease course as well as severity. Earlier we reviewed macrophage polarization in case of nonalcoholic fatty liver disease (NAFLD). Here we review it more comprehensively regarding crucial modes, of macrophage polarization, inflammatory, as well as non inflammatory which sees to the formation of insulin resistance (IR) as well as type 2 diabetes mellitus (T2DM). Details of macrophage polarization, bioenergetics macrophage adaptations in different scenario is discussed in detail along with role of transcription factors like interferon regulatory factor 5 (IRF5), nuclear factor kappa B (NFKB), toll like receptor 4 (TLR4), liver X receptor (LXR), activator protein 1(AP1), hypoxia inducible factor 1(HIF1), signal transducers and activators of transcription (STATS) in all these signaling besides peroxisome proliferator activated receptor (PPAR).
\end{abstract}

Volume 8 Issue I - 202 I

\author{
Kulvinder Kochar Kaur,' Gautam Allahbadia, ${ }^{2}$ \\ Mandeep Singh ${ }^{3}$ \\ 'Scientific Director, Dr Kulvinder Kaur Centre for Human \\ Reproduction, India \\ ${ }^{2}$ Scientific Director, Ex-Rotunda-A Centre for Human \\ Reproduction \\ ${ }^{3}$ Consultant Neurologist, Swami Satyanand Hospital, India
}

\begin{abstract}
Correspondence: Dr Kulvinder Kochar Kaur, M.D, Scientific Director, Dr Kulvinder Kaur Centre For Human Reproduction, 72 I, G.T.B. Nagar, Jalandhar- |4400I, Punjab, India, Tel 9|-|8|-950|358|80, Fax 9|-18|-46|3422 Email kulvinder.dr@gmail.com
\end{abstract}

Received: December 21, 2020 | Published: February 09, 2021

Keywords: diabesity, macrophage polarization, T2DM, IR, transcription factors, IRF5, NFKB, STATS

\begin{abstract}
Abbreviations: T2DM, type2 diabetes mellitus; NAFLD, nonalcoholic fatty liver disease; IR, insulin resistance; IRF5, interferon regulatory factor 5; NKFB, nuclear factor kappa B; TLR4, toll like receptor 4; LXR, liver X receptor; AP1, activator protein 1; HIF1, hypoxia inducible factor 1; STATS, signal transducers and activators of transcription; PPAR, per oxisome proliferator activated receptor; AT, adipose tissue; CVS, cardiovascular system; TNF- $\alpha$, tumor necrosis factor alpha; iNOS, inducible nitric oxide synthase; BM, bone marrow; ROS, reactive oxygen species; TGF- $\beta$, transforming growth factor beta; ATM, adipose tissue macrophages; HFD, high fat diet; PDGFR, platelet derived growth factor receptor; DOI, diet induced obesity; ILC, innate lymphoid cells; BAT, brown adipose tissue; CLS, crown like structures; AAM, alternatively activated macrophages; OPN, osteopontin; SVF, stromal vascular fraction; EE, energy expenditure; FI, food intake; CCR2, chemokine receptor 2; NASH, nonalcoholic steatohepapititis; HSC, hepatic stellate cells; VLDL, very low density lipoprotein; PDK, pyruvate dehydrogenase kinase; HK, hexosekinase; uPFK, ubiquitous isoform of phosphofructokinase; TCA, tricarboxylic acid; ETC, electron transport chain; LPS, lipopolysaccharide
\end{abstract}

\section{Introduction}

The incidence of obesity is increasing globally and with that the other comorbidities like type2 diabetes mellitus (T2DM). T2DM is a disease having double etiologies namely inflammatory, as well as metabolic. In the last 2 decades, inflammation has been escalatingly recognized to have significant role in enhancing risk of insulin resistance (IR) in addition to it getting visualized as an etiological starting point for metabolic failure. Various studies have tried to describe the kinetics among inflammation as well as IR, where certain reports document local IR as preceding inflammation, ${ }^{1}$ while others document occurrence of inflammation following IR. ${ }^{2}$ But blunting of inflammatory response has constantly been documented as being protective metabolically, ameliorating the formation of IR as well as T2DM. Hence inflammation is observed to be the deciding factor in loss of tolerance to metabolic imbalance. IR in liver, adipose tissue (AT) as well as skeletal muscle is initially confronted with a burst of activity in pancreas which sustain normal level of glycemia (the prediabetic stage).$^{3-5}$ Once this stage is prolonged as well as insulin synthesis cannot meet insulin demands any longer frank T2D forms that predisposes subjects to a lot of sequelae as well as comorbidities. These sequelae along with comorbidities are mainly hepatic as well as those implicating the cardiovascular system (CVS) in nature and have a direct relation to escalating inflammation, hyperglycemia in addition to it dyslipidaemia. Earlier we have reviewed the etiopathogenesis of both obesity as well as T2DM and grouped 2 as diabesity with the markedly escalating incidence and tried further to emphasize on the role of macrophages and aimed to find various ways of trying to treat both with different agents including natural agents like resveratrol, 
monoterpenes etc. ${ }^{6-23}$ Here we further deeply explore the role of macrophages in etioathophysiology of T2DM.

\section{Methods}

We conducted a systematic review utilizing the Pubmed search engine on macrophage polarization, along with transcription factors regulating it using the $\mathrm{MeSH}$ terms $\mathrm{T} 2 \mathrm{D}$; macrophage polarization; macrophage bioenergetic needs; metabolic adaptations; IRF5; STATS; PPAR's; AP1; LXR1; HIF1; Epigenetics from1970's till date.

\section{Results}

We found a total of 1751 articles out of which we selected 190 articles for this review. No Meta analyses were done.

\section{Metabolism and inflammation relationship}

This was initiated by Hotasmiglil as well as Spiegelman discovering escalated expression of proinflammatory cytokine tumor necrosis factor alpha (TNF- $\alpha$ ) in adipose tissue (AT) in case of obese rats models of obesity. ${ }^{24}$ Neutralization of TNF- $\alpha$ in these obese rats resulted in marked enhancement of glucose uptake in response to insulin. 10 yrs later macrophages were pointed to be the major source of TNF- $\alpha$ as well as other proinflammatory cytokine like IL-6 as well as inducible nitric oxide synthase (iNOS) in obesity. ${ }^{25}$ Further macrophages dramatically collect within AT the time of obesity, resulting in insulin resistance (IR). These early studies emphasized on the role of Inflammation along with metabolic failure related to IR as well as type2 diabetes (T2D) Figure 1. Following that a lot of importance has been laid on immune system as the main controller of metabolic homeostasis. Innate immune cells i.e. macrophages, reside in all metabolic tissues which coordinate glycemic homeostasis namely AT, liver as well as pancreas. Tissue-resident innate immune cells form a tissue particular immune niche, as well as every niche having its specificities to deal with microenvironmental signals. Of these innate immune cells, macrophages have been the ones that have been studied the maximum $(25 \% \text { of AT innate immune cells })^{26} 20$ $35 \%$ of nonparenchymal hepatic cells in the liver, ${ }^{27}$ and almost $90 \%$ of immune cells in pancreatic islets. ${ }^{28}$

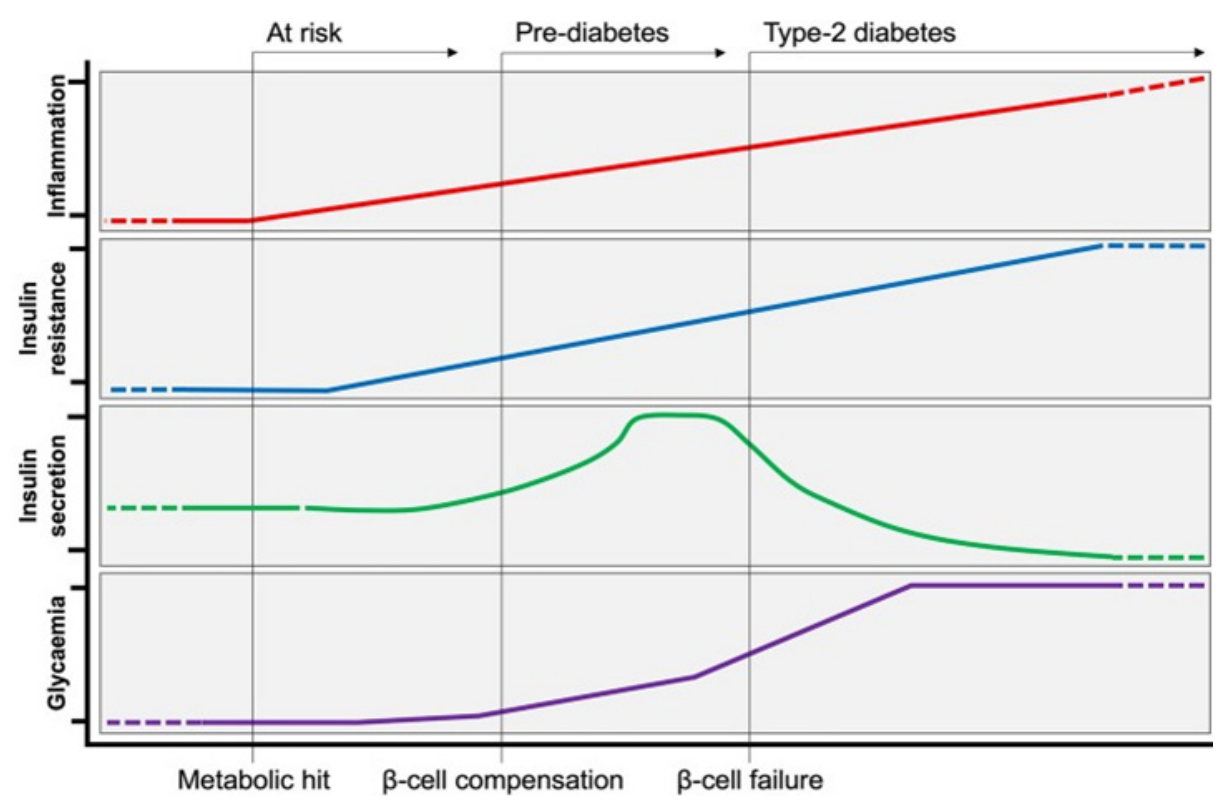

Figure I Evolution of Type-2 Diabetes. Following a metabolic hit, inflammation is at the initial steps of developing type-2 diabetes (T2D). Peripheral insulin resistance develops in tandem with increasing inflammation. Insulin resistance is initially met by a compensatory response from the pancreas, producing more insulin to maintain normoglycemia (pre-diabetes). Over time, insulin producing $\beta$-cells can no longer cope with increased demand, and insulin production ceases $\left(\beta\right.$-cell failure). At this stage of persistent hyperglycaemia T2D is established. ${ }^{189}$

\section{Polarization of macrophages-control of acute and chronic inflammation}

Initially macrophages got recognized by Metchinkoff as phagocytic cells. They constituted part of myeloid lineage with the capacity of rapid nonspecific responses for a wide variety of pathogens. Phagocytosis is a cellular method related to innate immune responses to pathogens, is key for the clearing of cellular debris, tissue repair, as well as manteinance of tissue homeostasis throughout the organism. These tissue-resident macrophages, form via progenitors within yolk sac, fetal liver as well as circulating monocytes which get origin via bone marrow (BM) ${ }^{29}$ During physiological circumstances, tissue-resident macrophages exert critical part in the integrity sustenance as well as homeostasis of the particular tissues. In response to environmental cues, macrophages, rapidly respond as well as thus adapt their function, they detect alterations in their microenvironment via cell surface receptor getting engaged. Basic receptors that convey environmental signals are toll like receptors (TLR's) that form part of the larger family of PRR. Ligating TLR's/ PRR by damage-associated molecular patterns/pathogen-associated molecular patterns (DAMP/PAMP) existing in the microenvironment stimulated transcriptional programs within macrophages to bring about an adapted functional phenotype..$^{30}$ These transcriptional modes have been well known, macrophages further adapt their cellular metabolism for meeting the bioenergic needs and get out maximum effector function. ${ }^{31}$ This has got a lot of limelight in current research. Currently the dichotomy utilized for telling the Polarization states of macrophages is like M1 is a proinflammatory or classically activated vis a vis M2 the anti-inflammatory or alternatively activated Figure 2. This knowledge comes via type 1 as well as type 2 immune responses 
that are canonically related to signaling molecules that get liberated on polarization. Macrophage signaling further polarizes the adaptive immune compartment for manteinance of chronic T helper (Th1/17 or Th2 response. M1 State correlates with type1 responses as well as synthesis of proinflammatory mediators correlated with bacterial or viral responses. M1 Macrophages possess robust microbicidal as well as AP capabilities. They synthesize proinflammatory cytokines that are very powerful like Tumor necrosis factor alpha (TNF- $\alpha$ ), Interleukin (IL-6), IL-1 $\beta$, as well as reactive oxygen species (ROS).

M2 Macrophages produce type 2 signaling, specifically in response to extracellular pathogens (helminthes, parasites), synthesizing anti-inflammatory cytokines like IL-10 as well as transforming growth factor beta (TGF- $\beta$ ). M2 Polarization is also thought of as a pro-resolution response that correlates with the latter parts of inflammation that is resolving. This adaptive immune system properly goes through Th2 Polarization that manufacture regulatory as well as remodeling cytokines like IL-4, IL-5 as well as IL-13. Hence the immunoregulatory response occurs secondary to specialized regulatory $\mathrm{T}$ cells (Treg) subpopulation. The pro-resolution effect can come out as scarring or tissue remodeling, which if abnormal lead to tissue fibrosis, type 2 effector molecules further also escalate allergic responses. ${ }^{32}$ Despite this classification, a continuing diverse response as well as macrophage phenotypes. Innovative functional classifications show Polarized Macrophages along a sliding scale between M1 as well as M2 based on their chemokine/cytokines liberation, transcriptional factor engagement as well as recently on the cellular metabolic phenotype. ${ }^{33}$ The increase of single cell sequencing as well as mass cytometry (CyTOF) have travelled a long way to help in divergence of functional separation as well as plasticity of Macrophages ${ }^{34}$ Figure 2 \& Figure 3.

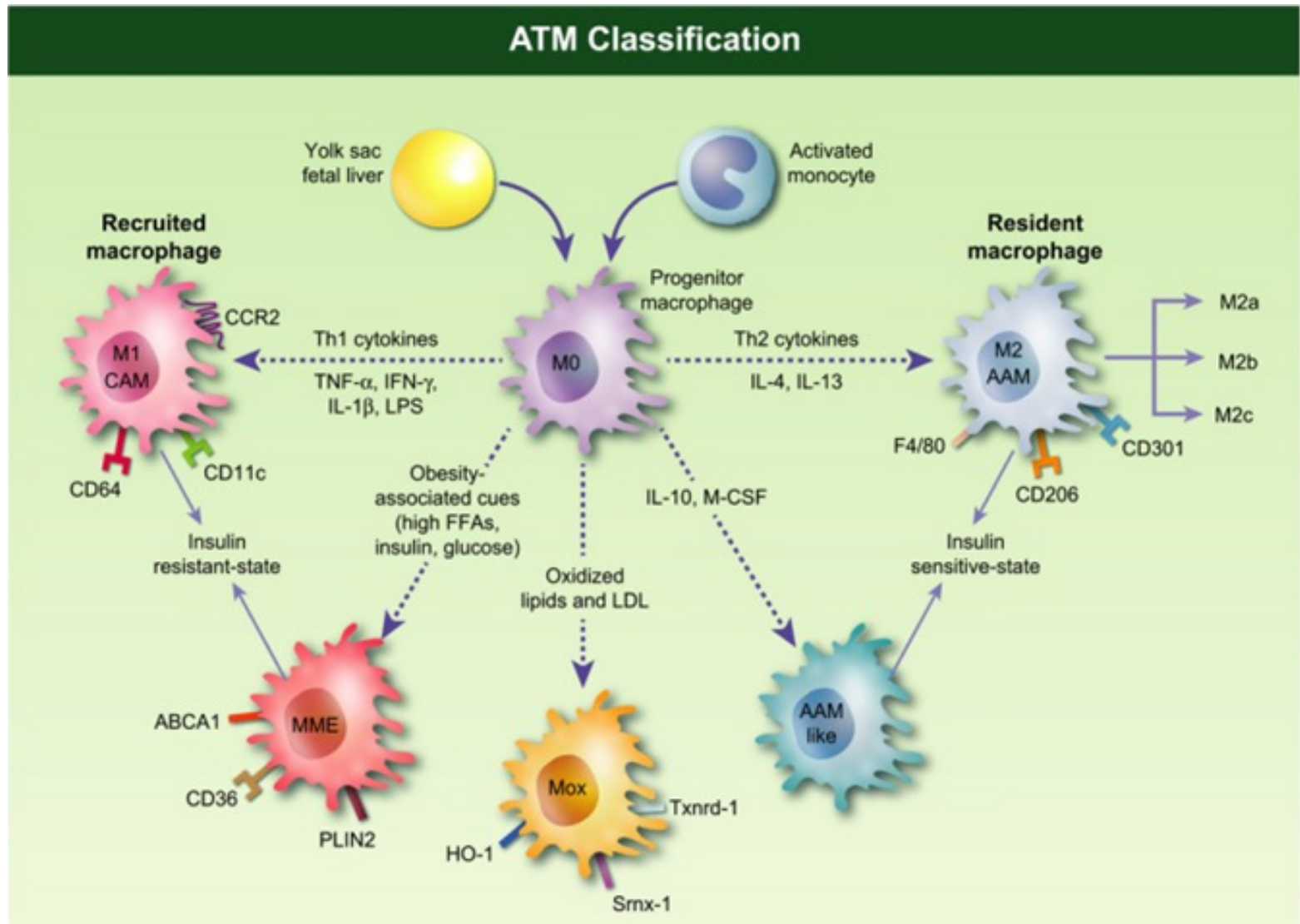

Figure 2 Adipose tissue macrophages (ATMs) classification. 'M0' macrophages originate from bone marrow-derived monocytes or yolk-sac progenitors. Depending on the stimuli, studies in mice have demonstrated that 'MO' macrophages differentiate into several subsets. Based on in vitro experiments, 'MI' or 'classical activated' macrophages (CAMs) originate from the stimulation with lipopolysaccharide (LPS), interferon (IFN)-c or tumor necrosis factor a (TNF-a). These macrophages are characterized by an IL- I 2high, IL-23high and IL-I Olow phenotype, display the surface marker CD I I C in addition to F4/80 and CD I I b, and produce pro-inflammatory mediators like TNF-a, IL-6, IL-Ib and nitric oxide (NO). 'M2' or 'alternatively activated' macrophages (AAMs) are macrophages with an anti-inflammatory phenotype derived from IL-4, IL-I0, IL-I3 and glucocorticoids stimulation. M2 macrophages exhibit an IL-IOhigh, IL-I 2 low and IL-23low phenotype, express the cell-surface markers CDI Ib, F4/80, CD30I and CD206, and secrete IL-4, IL-I0 and IL-I receptor antagonist (IL-IRa). 'M2' macrophages are further divided into three major variants: 'M2a', elicited by type II cytokines IL-4 or IL-I3; 'M2b', obtained by triggering of Fc gamma receptors in the presence of a Toll receptor stimulus; and 'M2c', elicited by glucocortocoids, IL-I0 or TGF-b. In obese setting, metabolic cues (e.g. free fatty acids, high insulin, high glucose, oxidized phospholipids, oxidized LDL) give rise to a population of metabolic activated (MMe) or oxidized (Mox) macrophages associated to an insulinresistant state. For instance, saturated fatty acids, which are released from hypertrophied adipocytes during the course of obesity, can act as a danger signal to MMe macrophages via the TLR4 complex. MMe macrophages exhibit a phenotype strikingly different from the typical MI/M2. Cell-surface proteins specifically overexpressed by MMe macrophages include ABCAI, CD36 and PLIN2, while conventional MI/M2 markers are suppressed. Mox macrophages are characterized by high expression of heme oxygenase-I (HO-I), sulforedoxin-I (Srnx-I), thioredoxin-I reductase (Txnrd-I), all redox-regulatory genes under the control of the Nrf2 transcription factor, IL-I 0 and VEGF. ${ }^{190}$ 


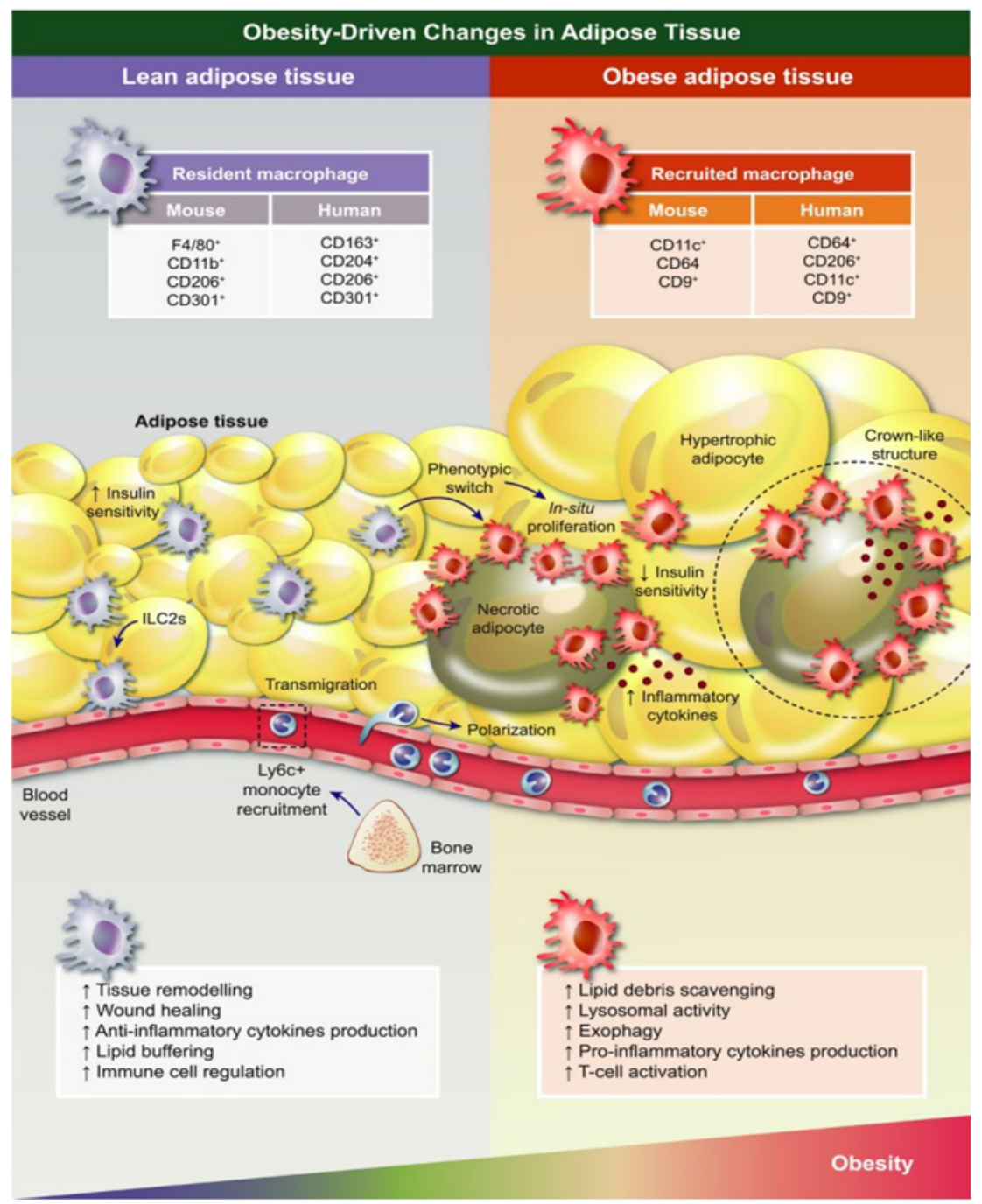

Figure 3 Obesity-driven changes in adipose tissue-associated macrophages (ATMs). In lean adipose tissue (AT), eosinophils and type 2 innate lymphoid cells (ILC2s) constitutively produce Th2 cytokines interleukin-4 (II-4) and interleukin-I 3 (II- I 3), respectively, which promote anti-inflammatory polarization of macrophages. As adiposity increases, obese AT manifests adipocyte hypertrophy, which leads to hypoxia, endoplasmic reticulum stress and lipotoxicity resulting in excessive leptin, cytokine and chemokine secretion. Among others, monocyte chemotactic protein-I (Mcp-I) induces Ly6C+ monocytes recruitment giving rise to tissue macrophages. Recruited macrophages, increased in number due to augmented infiltration and local proliferation, undergo a drastic change in distribution, forming crown-like structures (CLSs) around dead adipocytes. Additionally, they adopt a differential spectrum of functional properties and transcriptomic programming more prone to inflammation and metabolic dysregulation. This includes increased production of pro-inflammatory cytokines and other secretory products. ${ }^{190}$

\section{Metabolic physiology as well as pathology-role of tissue macrophages}

Good interaction among insulin signaling as well as insulin target tissues like pancreas, AT, liver as well as skeletal muscles are the ones responsible for sustenance of metabolic homeostasis following physiological challenges which cause transient difference in glycaemia, lipaemia like in fasting or feeding..$^{3-5}$ IR suggest transient or partial loss of interaction among tissues, when insulin target tissues get resistant to insulin signaling, even following early compensation via pancreas. T2D represents a stage when total loss or near loss of communication occurs when insulin synthesis does not meet body's needs of controlling glycaemia. All of these tissues have its own specific niche of macrophages having essential physiologic function that sustain integrity of tissues, adapting at every stage of T2D formation..$^{3-5,35,36}$ Tissue macrophages actions have been demonstrated to be markedly strong regulators of insulin signaling, their sensitivity as well as resistance Figure 4.

\section{Macrophages from pancreatic islets}

Pancreatic islets are the ones distributed throughout the exocrine pancreas; represent micro-organs necessary for systemic glucose homeostasis. Maximum of these Islets are composed of $\beta$ cells that respond to glucose within seconds by liberating proper levels of insulin needed for appropriate energy supply to insulin sensitive tissues. Innate immune cells form part of pancreatic Islets also. In case of steady state, Macrophages represent the instant immune cells in both mice as well as humans. ${ }^{28,37,38}$ Following greater than 20yrs of their finding, islets macrophages phenotype is still not known. Converse to adipose tissue macrophages (ATM) as well as liver macrophages islets macrophages don't stick to M1 as well as M2 polarization paradigm related to metabolic protection as well as dysfunction, respectively. Actually M1 indicators (like CD11c, MHC II) get constitutively expressed by macrophages on healthy pancreatic Islets, along with the high expression of IL- $1 \beta$, TNF- $\alpha$ as well as other proinflammatory transcription factor interferon regulatory factor (IRF)-5 in the 
same. . $8,37,39$ Further no expression of M2 pointers (CD206), as compared to stromal Macrophages of the exocrine pancreasis are seen. ${ }^{37}$ Role of islets macrophages with regard to islets homeostasis has only started to gain significance. Imaging done in situ showed that macrophages are very near to both $\beta$ cells as well as vasculature, in mice. ${ }^{40}$ Islets Macrophages are the ones that keep checking the $\beta$ cells insulin secretion relating to glucose by finding endogenous ATP which gets released along with insulin. ${ }^{41}$ Macrophages, in turn might directly stimulate or escalate insulin secretion via production of factors like retinoic acid. ${ }^{28}$ In contrast to any other tissues $\beta$ cells express the maximum IL-1 receptor 1 (IL-1R1)that markedly points to a physiological part of IL- $1 \beta$ in $\beta$ cells function. ${ }^{42}$ Now it is well known that that acute but not chronic exposure to IL- $1 \beta$ stimulates insulin secretion in mice as well as humans. ${ }^{43}$ Basic mechanism is not known, although might have a greater than insulin granule docking at the plasma membrane following escalated exocytosis..$^{43}$ For corroborating this posit using transgenic murine models $\beta$ cells targeted removal of IL-1R1 interferes with peripheral glucose tolerance through decreased glucose stimulated insulin secretion via 2 studies. ${ }^{44}$ Others show that feeding stimulates a physiological enhancement of circulating IL-1 $\beta$, that further escalates insulin secretion. ${ }^{45}$ It was said that peritoneal macrophages are the ones responsible for IL-1 $\beta$ secretion, that responded in response to glucose metabolism as well as bacterial products, liberated IL- $1 \beta$, which then in turn stimulated the $\beta$ cells. ${ }^{45}$ It is not yet been sure that probably Islets resident macrophages might also synthesize IL-1 $\beta$ Post prandially, actually these macrophages might turn out to be the major IL- $1 \beta$ source in Islets microenvironment. In to these findings prove that physiological IL- $1 \beta$ amounts have a key role in exaggerating insulin secretion.

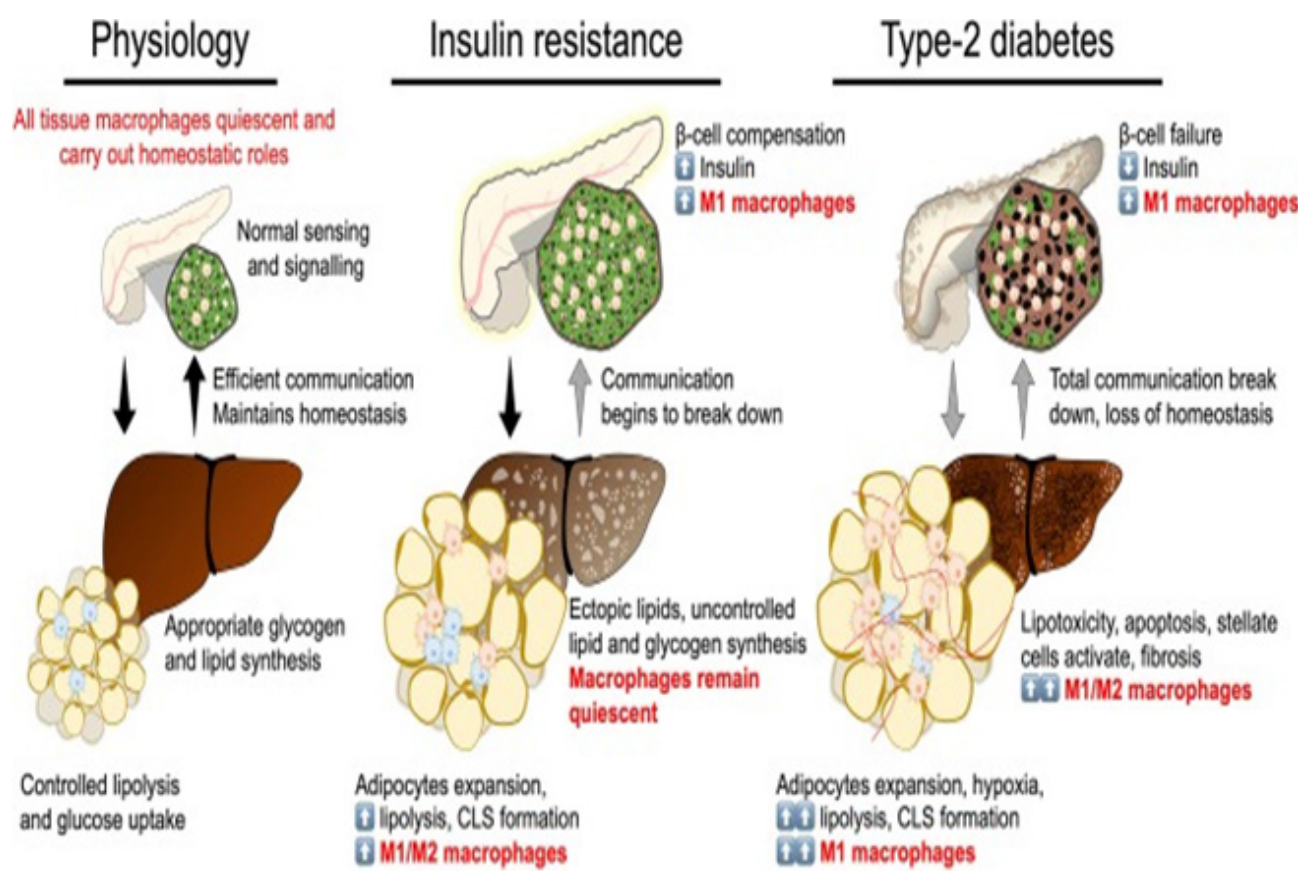

Figure 4 Break down of insulin secretion and sensitivity in type-2 diabetes. At the physiological state glycaemic homeostasis is maintained by efficient communication between the insulin secreting organ, the pancreas, and insulin target organs (adipose tissue and liver).All tissues are populated by their respective tissue macrophages that participate in maintaining tissue homeostasis and physiological function. Insulin resistance is a breakdown of communication at insulin target tissues. At the onset of insulin resistance, macrophages accumulate in adipose tissue and pancreatic islets. Crown-like structures develop in adipose tissue with heterogeneously polarized macrophages and a decrease in adipocyte insulin sensitivity. Pancreatic islet size increases with increased $\beta$-cell number and increased macrophages. Increase in $\beta$-cell number allows compensatory insulin release to overcome insulin resistance. Increasing insulin resistance and systemic inflammation result in $\beta$-cell failure when insulin secretion no longer compensates for resistance and persistent hyperglycaemia develops. At the stage of type- 2 diabetes, a complete breakdown in inter-organ communication occurs, insulin secretion drops and inflammatory macrophages permeate adipose tissue and the liver. Chronic inflammation, hyperglycaemia and dyslipidemia lead to the development of non-alcoholic steatohepatitis. ${ }^{189}$

Greater insulin synthesis is needed in case of obesity for manteinance of amounts of glucose. Due to this both the $\beta$ cells numbers as well as Islets size escalate, by local proliferation of earlier existent $\beta$ cells mainly Figure 3. Here in slow collection of macrophages might participate significantly in $\beta$ cells adapting to early rise of weight as well as insulin resistance (IR) formation .This way $\beta$ cells might allow $\beta$ cells mass expansion as well as needed angiogenesis in the 1st wk of high fat diet (HFD) as well as initial adapting of young $\mathrm{db}$./db mice. Actually in case of macrophages removed mice displayed less than $\beta$ cells proliferation rate, reduced insulin secretion as well as altered glucose tolerance as compared to controls. ${ }^{46}$ This enhancing of $\beta$ cells proliferation by islets macrophages is brought about by platelet derived growth factor receptor (PDGFR) signaling path. ${ }^{47}$ On obesity becoming chronic, ultimately insulin secretion does not compensate regarding enhanced insulin needs, ending in hyperglycemia as well as T2D.The $\beta$ cells failing correlates with local inflammation of the Islets as well as synthesis of inflammation effectors(IL-1 $\beta$, TNF- $\alpha$, CCL2).$^{38,42,47,48}$ Two separate subsets of macrophages have been isolated within the islets; resident macrophages as well as proinflammatory macrophages. Islet resident macrophages (CD11b+Ly6C- or F4/80 highCD11clow) are high at steady state while proinflammatory macrophages $(\mathrm{CD} 11 \mathrm{~b}+\mathrm{Ly} 6 \mathrm{C}+$ or F4/80lowCD11 chigh) collect in the course of obesity formation. ${ }^{47,48}$ Whereas CD11b+Ly6C+ macrophages get recruited via monocytes, the F4/80 lowCD11chighmacrophage replicate in situ. Regarding this chlodronate liposome macrophage removal rescues glucose stimulated insulin secretion in models of genetic as well as palmitate stimulated obesity in mice. ${ }^{32}$ Intriguingly even following escalation of islets macrophages amounts diet induced obesity (DIO) does not influence macrophages phenotype. ${ }^{37,47}$ Other source of inflammation 
factors which might take part in islets inflammation is endocrine cells themselves that has $\beta$ cells as well. Actually RNA seq of Islet cells from T2D patients showed an inflammation signature correlated with $\beta$ cells dysfunction relative to Islet cells from healthy controls, which was thought to be secondary to not only immune cells but to endocrine cells as well ,fuelling this local inflammation. ${ }^{49}$ These outcomes a little contradicting, point that islets macrophages are not the only ones causing inflammation of islets in obesity .Thus greater studies are needed for full definition of their phenotypes and to evaluate the role that immune cells might have like innate lymphoid cells (ILC) as well as their probable part in control of insulin secretion as well as $\beta$ cells mass expansion..$^{28}$

\section{Macrophages from AT-metabolic homeostasis}

Physiologically AT controls longtime energy stores, appetite via endocrine signaling as well as body temperature in case of brown adipose tissue (BAT). Adipose Tissue Macrophages (ATM), usually express an M2 profile during steady state during physiological conditions. They express mannose receptor CD206, CD301 along panmacrophage markers like F4/80 in mice, CD14 (in humans) CD68 as well as CD11b. ATM homeostasis signaling has expression of arginase (ARG1), IL-10, as well as other type2 effectors and catecholamines. The transcription factor PPAR-y is expressed greater in these cells as well as regulates oxidative metabolism ability to deal with a lipid-rich environment. In this niche ATM's crosstalk with other immune cells as well as give signals regarding stimulation as well as repression of $\mathrm{B}$ as well as T cells, neutrophils, NK cells, as well as ILCs. ${ }^{50}$ ATM's control tissue Homeostasis by removal of adipocytes that are in process of death as well as dead cells, the efferocytic mode sustains an anti-inflammatory environment. Actually murine AT that presents escalated dying adipocytes in view of targeted stimulation of caspase 8 have the property of enhanced number of alternately activated antiinflammatory macrophages (M2, CD206+), surrounding both dead as well as dying adipocytes. ${ }^{51}$ This group of cells surrounding adipocytes in a ring like structure is known as crown like structures (CLS). In lean AT CLS are occasional. During physiological changes, AT homeostasis gets challenged every day during feeding times, hence expansion as well as storage of lipids, or mobilization of lipids that are stored during fasting or cold exposure. Escalated lipid buffering ability is present in ATM's which aids in capturing of lipids liberated by dead adipocytes, even at the time of physiological conditions like weight loss, fasting stimulated lipolysis, ${ }^{52}$ or thermogenesis. ${ }^{53}$ Intriguingly, in case of obesity macrophages-related excess lipid capture controls systemic glucose tolerance. Lipids get stored within macrophages and get liberated into circulation in a regulated way ${ }^{54}$ Figure $2 \&$ Figure 3.

This lipid buffering ability of ATM's prevents ectopic lipid getting preserved, proinflammatory collection of lipids as well as systemic lipotoxicity/dyslipidaemia. Within M2 macrophages of AT lysosomal activity gets stimulated for dealing with environmental lipid overload. Intriguingly, inhibiting lysosomal biogenesis and hence lipid collection as well as catabolism within ATM's reduces adipocytes lipolysis. ${ }^{55}$ Innovative pathways of lipid liberation independent of canonical lipolysis have been shown. Adipocytes liberating exosomesized lipid enriched vesicles that have to be picked up as well as stored via ATM's. ${ }^{56}$ This lipid capture gets aided by ATM's expressing FA transporter (CD36) as well as lipid scavenger receptor MSRI. ${ }^{55}$ Most of insight regarding macrophages crosstalk with environmental lipids as well as their modes of stimulation have arrived via the atherosclerosis field as well as studying the foam cells. Actually initial work done by Nagy et al. ${ }^{57}$ emphasized on the significance of receptors like CD36, letting macrophages to internalize oxidized lipids that in turn work as nuclear receptor ligands (PPARy here). These Nagy et al., ${ }^{57}$ modes turned out to be the first to bring about connection between metabolic stress, transcriptional control as well as phenotypical plasticity. ATM's part in thermogenesis is a budding topic as well as pathways causing stimulation of ATM's are under evaluation. ${ }^{50} \mathrm{An}$ innovative population of macrophages having role in AT thermogenesis has been isolated; namely sympathetic neuron correlated macrophages (SAM) ${ }^{58}$ These SAM vary morphologically from ATM's as well as are located at the fibers of the sympathetic nervous system in AT. In contrast to ATM's, SAMs possess molecular apparatus for uptake as well as catabolism of norepinephrine that blunts catacholamine stimulated lipolysis. ATM's further possess part in iron homeostasis, where intracellular iron is a source of free radicals as well as cofactor for a lot of proteins. $25 \%$ of macrophages via lean AT are thought to be ferromagnetic.i.e. iron loaded, with this percentage decreasing with obesity. ${ }^{59}$ Recycling of iron via ATM's aids in AT homeostasis, where an up regulation of iron-associated genes takes place at the time of adipogenesis, with an extra iron causing adipocytes IR. ${ }^{60}$

In adipogenesis, a greater direct part is played via ATM's, where alternatively activated macrophages (AAM) via a niche for the formation of adipocytes as well as in vascularization of AT. ${ }^{61}$ The collection of M2 ATM's in the CLS surrounding dead adipocytes results in the pre adipocytes recruitment via response to osteopontin (OPN). But recently it was shown that M2 like ATM's inhibit the replication of adipocytes progenitors via TGF $\beta$ signaling. A significant study via Bourlier et al., ${ }^{61}$ showed that subcutaneous ATM's are the predominantly $\mathrm{CD} 206+$ ), being a main source of matrix degrading enzymes, and thus they become main source for tissue remodeling. Secreted factors via ATM's were observed to be aiding angiogenesis as well as inhibit adipogenesis in stromal vascular fraction (SVF) progenitor cells. ${ }^{61}$ Regulation of angiogenesis is crucial in sustenance of tissue homeostasis since it limits hypoxic sites and ensures proper irrigation giving nutrients as well as $\mathrm{O} 2$ to the microenvironment. This work displays that ATM's are basically protective as well as in early stages of caloric overload, aid in coordinating AT adaptation. ${ }^{61}$ Lastly AAM have the property of synthesizing IL-10, an anti-inflammatory cytokine significant for modulation of insulin sensitivity. ${ }^{62}$ Actually IL-10 therapy enhances global insulin sensitivity in vivo ${ }^{63}$ as well as its expression is positively associated with insulin sensitivity in humans. ${ }^{64}$ Intriguingly haematopoietic removal of IL-10 does not promote obesity nor are IR, pointing that other factors as well as pathways there for manteinance of metabolic health. ${ }^{65}$ Moreover ATM's can liberate exosome possessing miRNA like miR155, which controls insulin sensitivity. These ATM's derived exosome from lean mice improve glucose intolerance as well as insulin sensitivity when given to obese mice. ${ }^{66}$

\section{Metabolic inflammation-AT macrophages}

There is complicated etiopathology of obesity that further becomes etiological regarding IR as well as T2D. Basic cause of obesity is chronic imbalance among energy expenditure (EE) as well as food intake (FI) that causes low grade inflammation. Chronic low grade inflammation is the one mostly detailed as Meta inflammation, initiating point of whom lies in ATM. In obesity collection of inflammatory ATM takes place in obesity, having a critical part in the pathogenesis of obesity stimulated $\operatorname{IR}^{24,25}$ Figure 4. Inflammatory ATM's are equivalent to M1 macrophages and isolated as $\mathrm{F} 4 / 80+\mathrm{CD} 11 \mathrm{~b}+$ cells , that are also positive for CD11c as well as overexpress IL-6, TNF- $\alpha$, iNOS as well as C-C chemokine receptor 2 (CCR2). ATM accumulation occurs during obesity initially due to in situ replication at CLS, followed by recruitment of circulating monocytes which differentiate into inflammatory macrophages ${ }^{67}$ First proliferative phase is secondary to IL-4 signaling via signal transducer and activator of transcription of (STAT)-6. Infiltrating Macrophages escalate on CCL2 signaling to monocytes. Variety of studies has 
shown the significance of CCR2/ CCL2axis in getting circulating monocytes recruited. ${ }^{68}$ Further migratory ability of Macrophages gets affected by obesity. Actually, Netrin-1, a laminin associated molecule which we know has chemoattractant/repulsive characteristics, gets stimulated via palmitate .It inhibits ATM's migratory ability towards lymph nodes and hence aids in ATM's collection in situ. ${ }^{69}$ The lipid buffering ability of ATM's in early dysmetabolism is advantageous since it escalates lysosomal program related to M2 macrophages. ${ }^{4-6}$ the excess of lipids within ATM's influences their polarization towards an M1 phenotype. ${ }^{70}$ Single cell transcriptomics methods corroborate the heterogeneity of the ATM's that finds 3 kinds of macrophage populations within obese AT. Resident macrophages (F4/801ow) expressing CD206 are sustained in obese AT while Ly6C expression is the property of newly recruited macrophages (also F4/80Hi). The proinflammatory subset containing lipid laden macrophages in CLS possess the property of expressing CD $9^{71}$ Jaitin et al., ${ }^{26}$ corroborated the phenotype as well as presence of CD 9+ lipid laden macrophages at the CLS. As per them CD 9+cells prevent inflammation as well as adipocytes hypertrophy through the lipid receptor TREM $2 .{ }^{8}$ By proteomic evaluation also found particular ATM markers stimulated via stimuli that reproduced the AT microenvironment via palmitate, insulin, as well as high amounts of glucose. ${ }^{72}$ This activation of ATM's produces metabolically activated macrophages (Mme), that is both function as well as phenotypically separate from classically activated M1 macrophages. The significance of proinflammatory ability of the newly recruited ATM's in the formation of obesity is well known. Activated macrophages surround dead adipocytes and fuse resulting in development of multinucleated giant cells,${ }^{73}$ which stands out with chronic inflammation as a hall mark as well as is associated with IR. ${ }^{74}$ Ablating CD11c+cells at the time of obesity was demonstrated by Patsouris et al. ${ }^{75}$ to restore insulin sensitivity by reduction of inflammation markers. ${ }^{75}$ Interferon regulatory factor 5(IRF5) represents a proinflammatory transcription factor, mostly limited to $\mathrm{CD} 11 \mathrm{c}+$ cells , that stimulates macrophages Polarization towards M1 phenotype $^{76}$ as well as is specifically induced within ATM's in diet induced obesity (DIO). ${ }^{77,78}$

\section{Macrophages from liver-metabolic homeostasis}

Resident macrophages in Liver, also known as Kupffer cells (KC's) constitute upto $80-90 \%$ of total body macrophages population with the property of expression of canonical macrophages markers (F4/80, CD14, CD68, CD11b) along with C type Lectin (Clec)-4F. ${ }^{5}$ Clec-4F represents a marker of genuine $\mathrm{KC}$ 's which are functionally separate, specific as well as self-renewing resident macrophages. ${ }^{79}$ KC's are part of the Reticulo Endothelial system of the live, present near the blood vessel's within lumen of hepatic sinusoids, along with regulate proliferation besides apoptosis on injury in addition to at steady state they clear blood of old erythrocytes as well as recycle iron by degradation of Haemoglobin. ${ }^{80}$ Their presence is as per adapting to their role in clearing portal blood of pathogens, microorganisms, as well as cellular debris ${ }^{81}$ They select as well as remove debris through blood via scavenger receptors as well as canonical PRR's present on the cell surface. Significantly, KC's put immune tolerance in the liver, an organ that is all the time exposed to antigen's as well as bacterial endotoxins via intestine In addition to portal blood. KC's maintain anti-inflammatory environment by different ways: liberation of IL10, low expression of MHC-II as well as high expression of PD1, that limits antigen Potentiation ability with a strong inhibition of Tcells response. ${ }^{80}$ Intriguingly even on IFNy priming, KC's stimulate different types of Tregs by specific immunoregulatory subset of Tcells which sustain immune tolerance. ${ }^{82}$ At steady state KC's have minimal crosstalk with distant non immune cells since they are not typically motile cells. On need of micro environmental inter communication;
KC's liberate cytokines for signaling monocytes to differentiate into macrophages. ${ }^{80}$

\section{Metabolic inflammation-liver macrophages}

Once there is extension of inflammation via AT it results in escalation of proinflammatory mediators within the circulation and thus escalation in obesity. IR, constant glucolipotoxicity as well as systemic inflammation go parallel with ectopic lipid fat accumulation, main site of that being liver. In cases of obesity as well as T2D varying changes take place in liver extending from benign steatosis to fibrosis as well as cirrhosis $^{83}$ reviewed. ${ }^{23}$ These are referred to as nonalcoholic fatty liver disease (NAFLD), in which this lipotoxicity, inflammation as well as fibrosis represent the greater than advanced stages of nonalcoholic steatohepapititis (NASH). Role of liver macrophages, KC's are crucial in the propagation of NASH, in view of their proapoptotics as well as proinflammatory responses towards lipotoxic hepatocytes as well as their ability of activation of matrix producing hepatic stellate cells (HSC's). This ectopic lipid fat accumulation stimulates activation of immune cells as well as an inflammatory environment that stimulates IR. Intriguingly, when a nonbiased transcriptomic signature evaluation was done, no variations as far as expression of genes correlated with proinflammatory signatures, among liver Macrophages via lean as well as obese (same data obtained from mice fed 9 wks of high fat diet (HFD). Metabolic alterations do not correlate with a proinflammatory stimulation of liver macrophages ${ }^{84}$ But the transcriptomic signature varies among different benign steatosis stage to that of NASH. During the conversion from steatosis to NASH, liver macrophages attack lipotoxic hepatocytes and thus stimulation of apoptosis as well as signal to HSC's for stimulation of their activation. ${ }^{83}$ Chronically damage to liver will end in fibrosis with extensive scarring response to dead or dying hepatocytes, with continuing fibrosis influencing liver function. ${ }^{83}$ At the NASH stage liver Macrophages pool is markedly heterogenous, with M1 like macrophages stimulating hepatocyte apoptosis, while M2 like macrophages aid in HSC's activation as well as fibrogenesis. ${ }^{83,85}$ The pro inflammatory transcription factor Interferon regulatory factor 5 (IRF5) has been demonstrated to have a key part in liver macrophages, that brings about the shift from benign steatosis to NASH. Hepatoprotection occurs once IRF5 expression is blunted via early up regulation of anti-apoptotic as well as immunoregulatory signaling, escalating Treg differentiation as well as IL-10 liberation on hepatocellular stress. ${ }^{85}$ Current research is trying to get insight into potential non inflammatory or no immune signaling efferent from KC's, especially effector molecules like insulin like growth factor binding protein -7 (IGFBP-7), controls insulin sensitivity in relation to obesity. ${ }^{86}$

\section{Development as well as manteinance of macrophages polarization in T2D}

Currently trying to define extracellular metabolic as well as molecular signals related to macrophages Polarization in metabolic inflammation as well as IR, is being actively worked .The Candidate metabolic immunogens that might be candidates are lipids, hypoxia, cell death as well as stress. ${ }^{52,73,87}$ In genetically obese mice $90 \%$ of adipose tissue macrophages (ATM's) surround dead adipocytes in fat depots, ${ }^{88}$ that points that dead adipocytes are the ones that contribute to damage associated molecular patterns (DAMP's) which results in crown like structures (CLS) development and/or collection of ATM's. Further hypoxic areas exist in obese AT along with expression of hypoxia related genes, that include hypoxia inducible factor 1 alpha (HIF 1- $\alpha$ ). This transcription factor further facilitates the proinflammatory abilities of ATM's in relation to obesity ${ }^{89}$ Moreover, lipolytic products as well generally lipids whose levels are escalated 
in obesity are very good candidates for stimulation of inflammatory response in ATM's. Nutritional Fatty acids activate TLR-4 in Macrophages which states signaling pathways. ${ }^{90}$ Triglyceride-rich lipids like palmitate or very low density lipoprotein (VLDL) activate macrophages, those up regulate intracellular amounts of ceramides and further accelerate proinflammatory responses. ${ }^{91}$ These modes activate NLRP3ASC inflassome that states caspase 1 modulated cleavage of pro IL-1 $\beta$ as well as pro IL-18 into their active states. Intriguingly saturated Fatty acids like palmitate have been demonstrated to activate the NLRP3 inflassome via an AMPK-autophagymitochondrial-ROS signaling axis, resulting in liberation of IL- $1 \beta$ as well as IL- $18 .^{92}$ Notably this IL-1 $\beta$ liberation by itself correlates with insulin resistance. Actually IL-1 $\beta$ avoids signaling via Tumor necrosis factor alpha dependent as well as in dependent modes. ${ }^{93} \mathrm{On}$ getting established these proinflammatory environmental factor, the formation of proinflammatory cytokines which recruit monocytes as well as other immune cells which maintain low grade chronic inflammation. Proinflammatory cytokines represent crucial factors in disrupting insulin signaling resulting in IR. ${ }^{94}$ They work via paracrine modes on insulin sensitive cells like adipocytes. Physiologically, On insulin binding to its receptor the phosphorylation of tyrosine residues of insulin receptor substrate 1 (IRS1) activates intercellular signaling pathways bringing about insulin action. ${ }^{95}$ With regards to metabolic inflammation, JNK1 as well as IKK have the ability of influencing insulin signaling by phosphorylation of inhibitory serine /threonine residues of IRS1,thus disrupting insulin signaling. ${ }^{96}$ On the same hand, pathways involving JNK1 as well as IKK might be activated via binding of fatty acids (FA's) to TLR's. Further IL- $1 \beta$ that also signals via IKK $\beta$ as well as $\mathrm{NF \kappa B}$, stimulates IR by repressing IRS1 expression at both transcriptional as well as post transcriptional levels. ${ }^{97}$ IL-6 signaling inhibits insulin sensitivity via separate modes that involve JAK-STAT pathway which regulates the transcription of its own suppressor, called suppressors of cytokine signaling (SOCS3). High amounts IL-6 stimulate greater than expression of SOCS3 that physically cross acts with tyrosine phosphorylated residues and this inhibits IRS1 binding to the insulin receptor. ${ }^{98}$

\section{Macrophages polarization-metabolic modes}

Just like any other cell Macrophages possess their own Metabolic needs and are dependent on similar bioenergetic pathways like the no immune cells-which are widely divided into glycolytic or mitochondrial Figure 5. Besides proinflammatory signaling as well as transcriptional regulation, cellular metabolism is getting recognized for its crucial role that it has in macrophages terminal differentiation. Metabolic pathways mobilization besides producing energy, further influences the degree of Macrophages effector function..$^{13}$ Initial studies on immunometabolism gave basic modes that fuel Macrophage in models systems having canonical activators. These studies aided in proper correlation of bioenergetic profiles to polarization status. Recent work is further working on this basis by evaluation of bioenergetic profiles as well as metabolic adaptations of tissue particular macrophage niches under both Physiological as well as pathological scenario and in response to varied stimuli. Funnily, the metabolic classification of macrophages was initially done with the 1st seeing that M2 macrophages were able to metabolize arginine. ${ }^{99}$

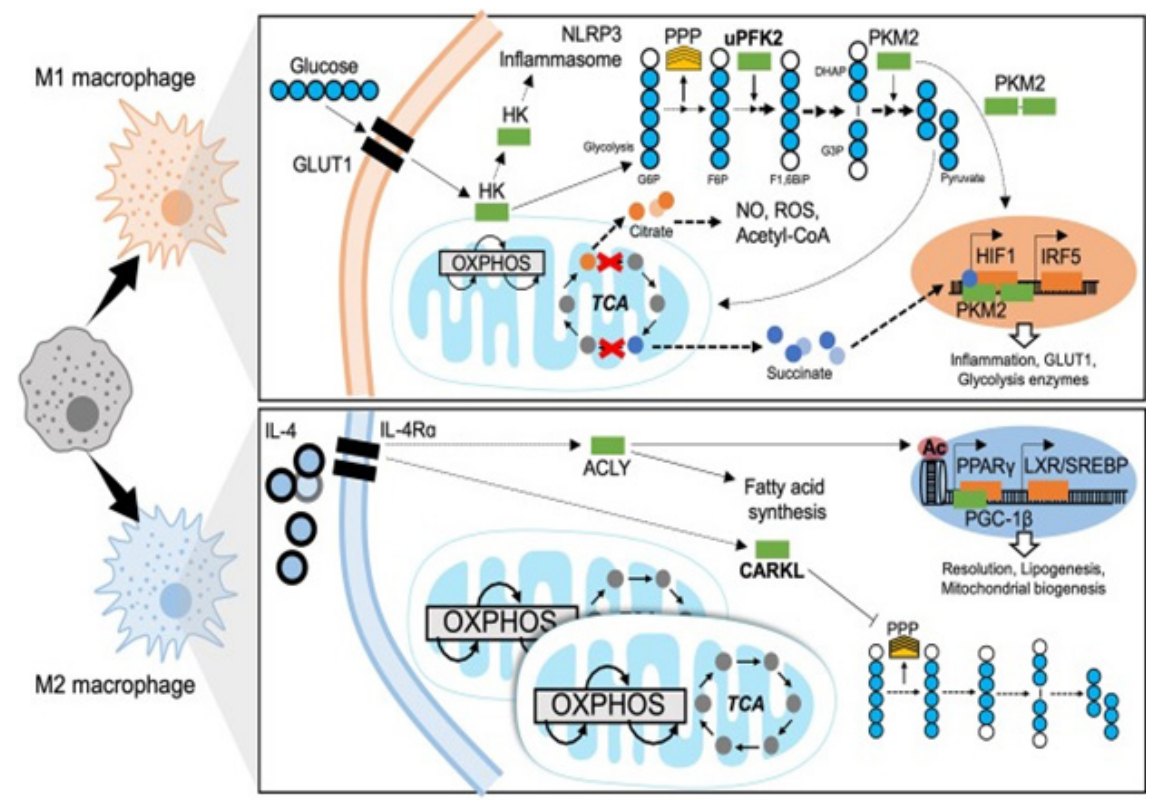

Figure 5 Metabolic mechanisms of macrophage polarization. MI macrophages are characterized by predominantly glycolytic metabolism. Glycolysis consists of breaking down a 6-carbon glucose molecule (where each carbon is depicted as a blue circle, white when phosphorylated) into 3-carbon sugars then into pyruvate, ATP, NADH, and $\mathrm{H}+$. The transcriptional programme that supports glycolysis is mediated by HIFI and at least in part by IRF5. A Glucose substrate is provided by increased expression of the glucose transporter GLUTI. Meanwhile several glycolytic enzymes undertake non-canonical roles to support MI effector functions. The mitochondrial tricarboxylic acid (TCA) cycle is disrupted, leading to accumulation of citrate and succinate which also enhance MI effector function. The M2 macrophage has a fully intact TCA cycle, enhanced OXPHOS and increased mitochondrial biogenesis. ATP citrate lyase (ACLY) is activated downstream of IL4 signaling and enhances M2 effector functions through epigenetic mechanisms and producing substrates for lipogenesis. The sedoheptulose kinase (CARKL) represses the pentose phosphate pathway (PPP). Transcriptional programmes for M2 macrophage metabolism are mediated by PPAR $\gamma$ and LXR. GLUTI, Glucose transporter-I; HK, Hexokinase; NLRP3, NACHT, LRR, and PYD domains-containing protein; OXPHOS, oxidative phosphorylation; TCA, tricarboxylic acid cycle; PPP, pentose phosphate pathway; uPFK2, ubiquitous phosphofructokinase2; PKM2, pyruvate kinase isozyme 2; G6P, glucose-6-phosphate; F6P, fructose-6-phosphate; FI,6BiP, Fructose-1,6-biphosphate; G3P, glyceraldehyde-3-phosphate; DHAP, dihydroxyacetone phosphate; NO, nitrous oxide; ROS, reactive oxygen species; CoA, Coenzyme A; HIFI, hypoxia-inducible factor I; IRF5, interferon regulatory factor 5; IL-4, interleukin 4; IL-4R $\alpha$, IL-4 receptor alpha; ACLY,ATP-citrate lyase; CARKL, carbohydrate kinase like/sedoheptulose kinase;Ac, acetylation mark; PPAR $\gamma$, peroxisome proliferator-activated receptor gamma; LXR, liver X receptor; SREBP, sterol regulatory element binding protein; PGC-I $\beta$, PPAR $\gamma$ coactivator I-beta. ${ }^{189}$

Citation: Kaur KK, Allahbadia G, Singh M. Targeting macrophage polarization for therapy of diabesity-the feasibility of early improvement of insulin sensitivity and insulin resistance-a comprehensive systematic review. J Diab Metab Disorder Control. 2021;8(I):6-25. DOI: 10.15406/jdmdc.2021.08.00216 


\section{Proinflammatory macrophages-metabolic adaptations}

The increase in glycolytic activity of proinflammatory macrophages was seen a lot of decades back, ${ }^{100}$ although the insight into the mode as well as its physiological importance have been only recently clarified. This has great importance in Macrophages Polarization towards an M1 phenotype Figure 4. The metabolic pathway that results in glucose converted to pyruvate is glycolysis via 10sequential enzyme catalyzed reactions. From this pathway ATP as well as NADPH gets synthesized. Glycolytic Metabolism promotes proinflammatory differentiation to aid in effective bacterial killing ${ }^{101}$ as well as liberation of proinflammatory mediators. Experimentally preventing glycolysis utilizing 2Deoxyglucose (2DG) prevents the proinflammatory macrophages response to LPS. ${ }^{102}$ Fast induction of glycolysis gets escalated by up regulation of glucose transporter 1(GLUT 1)-expression. ${ }^{103}$ This shift toward glycolytic metabolism is based on the transcription factor HIF $1-\alpha .{ }^{104}$ Its stability in hypoxic situations facilitates anaerobic metabolism along with escalated transcription of genes that code for glycolytic enzymes like, pyruvate dehydrogenase kinase (PDK) and hexosekinase (HK) that catalyzes glucose phosphorylation. Ubiquitous isoform of phosphofructokinase (uPFK) gets induced in M1 Macrophages by an HIF 1- $\alpha$ independent mode. Although uPFK is a greater than active form of PFK 2, its induction escalates glycolytic flux and facilitates the synthesis of fructose 2, 6P2 that allosterically activates PFK1, the enzyme s catalyzing for glycolysis. ${ }^{105}$ Besides hypoxia inducible factor 1-alpha (HIF 1- $\alpha$ ) dependent modes, research of IRF5-risk variants document that gain of function single nucleotide polymorphisms (SNP's) of IRF5 (related to autoimmune diseases) escalated glycolysis as well as inflammatory signaling, basally as well as in response to LPS. ${ }^{106}$ Certain glycolytic enzymes possess noncanonical parts in macrophages. Importantly pyruvate kinase is enzyme 2 (PKM2), that gets induced via LPS ${ }^{107}$ is present in the form of a dimer. This dimer might translocate towards nuclei and work as a cofactor for HIF1 Figure 4. Thus PKM2 takes part in a positive feedback loop with up regulation of proinflammatory as well as glycolytic genes secondary to HIF1 M1 activation. ${ }^{108}$ Further HK1 can get inhibited via bacterial products as well as subsequently dissociate from mitochondria, that activates the NLRP3 inflassome as well as a downstream formation of Proinflammatory cytokines. ${ }^{109}$ Although mode of resolving of glycolytic programming have not been worked out, a recent work by Ip et al., ${ }^{110}$ showed that IL-10 signaling causes anti-inflammatory actions via inhibition of translocation of GLUT 1 towards the membrane. ${ }^{110}$ Besides being a substrate for glycolysis, glucose also fuels the pentose phosphate pathway (PPP), needed for manufacture of nucleotides as well as NADPH meant for ROS synthesis via NADPH oxidase. On LPS stimulation as well as M1 polarization also induction of PPP occurs. ${ }^{111}$

The tricarboxylic acid (TCA)/Krebs cycle represent a mitochondrial metabolic pathway that aids in ATP synthesis and thus provide substrates for the electron transport chain (ETC) which supports oxidative phosphorylation (OXPHOS) Figure 4. Regarding proinflammatory macrophages, this Krebs Cycle gets disrupted at 2 crucial steps i) the collection of citrate because of reduction in isocitrate lyase and ii) the collection of succinate. Citrate efflux from mitochondria gets escalated in M1 macrophages. The collection of citrate has functional importance for inflammatory polarization, being needed for the synthesis of reactive oxygen species (ROS), NO, as well as prostaglandins. ${ }^{112}$ Citrate further acts as a substrate for conversion into acetyl CoA, thus, feeding fatty acid synthase (FAS) via ATP citrate lyase (ACLY) ${ }^{113}$ Inhibition of FAS via silencing of FAS in myeloid cells ,has been demonstrated to have protection against diet induced IR that blocks recruitment of ATM as well as chronic inflammation in mice. Here lies the importance of lipid metabolism in the Polarization as well as function of macrophages, as well as manufacture along with composition of the plasma membrane. ${ }^{114}$ Ultimately the collection of citrate results in reduction in the amounts of cisacotinate that is the precursor of itaconate that has been a well detailed anti-inflammatory intermediate. Itaconate's anti-inflammatory actions get exerted via inhibition of succinate dehydrogenase (SDH), ROS synthesis, as well as liberation of proinflammatory cytokines. An itaconate negative feedback loop has been detailed regarding lipopolysaccharide (LPS) as well as IFNy stimulation where itaconate shut down the inflammatory response. ${ }^{115}$ Conversely succinate accumulation facilitates SDH action as well as synthesis of mitochondrial ROS. ${ }^{116}$ Succinate might trigger the expression of IL-1 $\beta$ via stabilization of HIF $1 \alpha .{ }^{117}$ Hence proinflammatory macrophages have the properties of rise in glycolytic activity as well as reduction in OXPHOS. Acute LPS treatment causes a burst of oxidative metabolism in macrophages that enhances the pool of acetyl Co A that becomes available. This corroborates histone acetylation as well as downstream transcription of proinflammatory genes. ${ }^{118}$ Shutting down of oxidative metabolism, that is a hallmark of M1 macrophages, takes place after prolonged LPS treatments. Lastly, amino acids, the immunometabolism of which is relatively little known can also get mobilized and effect macrophages polarization. Like glutamine catabolism feeds the tricarboxylic acid (TCA) Cycle by producing alpha-ketoglutarate that acts as a cofactor for histone modifying enzymes that are involved in macrophage differentiation. ${ }^{119}$ Arginine is also metabolized to L-citrulline as well as a NO by iNOS that facilitates killing of bacteria.

\section{Anti-inflammatory macrophages-metabolic adaptations}

Mitochondrial respiration predominates in the M2 polarized state. M2 macrophages possess intact, totally functional TCA Cycle with escalated OXPHOS Figure 4. Both mitochondrial biogenesis as well as fatty acid oxidation (FAO) are escalated in a PPARy-coactivator$1 \beta$ (PGC-1 $\beta$ )-dependent method. ${ }^{120}$ As FAO is the basic source for substrates, glycolysis fuelled OXPHOS is not needed for manteinance of M2 macrophages. ${ }^{121}$ The molecular mode that connects metabolic adaptations of M2 macrophages to their functions in tissue homeostasis are mostly not evaluated. Intriguingly IL-4 activates ACLY, escalating substrate formation for histone acetylation. This epigenetic modification allows the transcription of specific M2 genes. ${ }^{122}$ Other probable modes involve the carbohydrate kinase like protein (CARKL) a sedoheptulose kinase which controls PPP. CARKL gets down regulated in response to LPS as well as expressed greater with IL-4 stimulation. ${ }^{123}$ CARKL activity inhibits the PPP in the M2 state Figure 5. Glutamine Metabolism further has a big part in M2 Polarization. With IL-4 stimulation expression of Slc1a5, that is a glutamine transporter, gets escalated. ${ }^{224}$ Glutamine catabolism besides glucose metabolism, results in development of UDP-GlcNAc which supports $\mathrm{N}$ glycosylation, a process needed for the expression of variety of M2 markers. ${ }^{125}$ Lipid synthesis that gets modulated by LXR is in centre of M2 effector function as well as resolution of inflammation. ${ }^{126}$ On Proinflammatory activation, LXR dependent lipogenesis gets inhibited. Since LXR is a prolipogenic nuclear receptor as well as transcription factor, it later involves SREBP1 to modulate the formation of anti-inflammatory lipids like eicosanoids, resolve's. ${ }^{127}$

\section{Analyzing metabolic adaptations of resident macrophages of tissues as well as insulin resistance}

The basic findings regarding macrophage bioenergetics were mostly determined with use of ex vivo modeling systems (like murine bone 
marrow (BM) or human monocyte derived Macrophages) as well as in response to known polarizing agents. Although they are applicable to most of macrophages, typically infiltrating macrophages responses towards complicated metabolic stimuli as well as heterogeneity of tissues resident macrophages needs to be worked out. Tissues resident macrophages face a competition regarding nutrients, with normoxic In addition to hypoxic areas besides crosstalk with other cells. They produce a response to complicated stimuli instead of unique stimuli. Bioenergetics adaptations of Tissues resident macrophages in obesity as well as IR have to be still worked out. Significantly, ATM's of obesity possess a unique hypermetabolic profile with both escalated glycolysis as well as OXPHOS as compared to lean ATM's, while sustaining a proinflammatory phenotype. ${ }^{128}$ More specifically, the proinflammatory ability of the obese ATM's is modulated via glycolysis independent of HIF $1 \alpha^{128}$ This bioenergetic profile is also separate from peritoneal macrophages, inspite of sharing of systemic glucolipotoxicity that is brought in by obesity. These findings underlie the specificity of metabolically activated macrophages as well as ATM's. Hypoxic areas form within AT on inappropriate expansion in obesity as well as IR. Hypoxia as well as imperfect angiogenesis are luring modes causing macrophage metabolic activation as well as their inflammatory Polarization. On the other hand the excess of free fatty acids (FFA) or lipolytic products in AT makes for a nutrient/ substrates-rich micro environment. The action of this lipid loading on macrophage metabolism as well as polarization needs to be evaluated under hypercaloric situations. Like the action of obesity on macrophage glutamine metabolism needs to be examined. Glutaminolysis is reduced in the $\mathrm{AT}$ of obese subjects as compared to lean ones as well as glutamine amounts in serum are reduced in patients having obesity or diabetes, pointing that there is an influencing part of glutamine metabolism in ATM's Polarization. ${ }^{129}$

\section{Macrophage polarization-transcriptional regulation}

Transcriptional Regulation regarding Macrophage Polarization has been well examined downstream of toll like receptor (TLR) ligation. Excellent studies found major TLR ligands along with critical transcription factors which modulate inflammatory responses. A lot of these pathways have been evaluated in metabolic diseases and represent crucial mediators of macrophage activation in obesity; IR as well as T2D Figure 6.

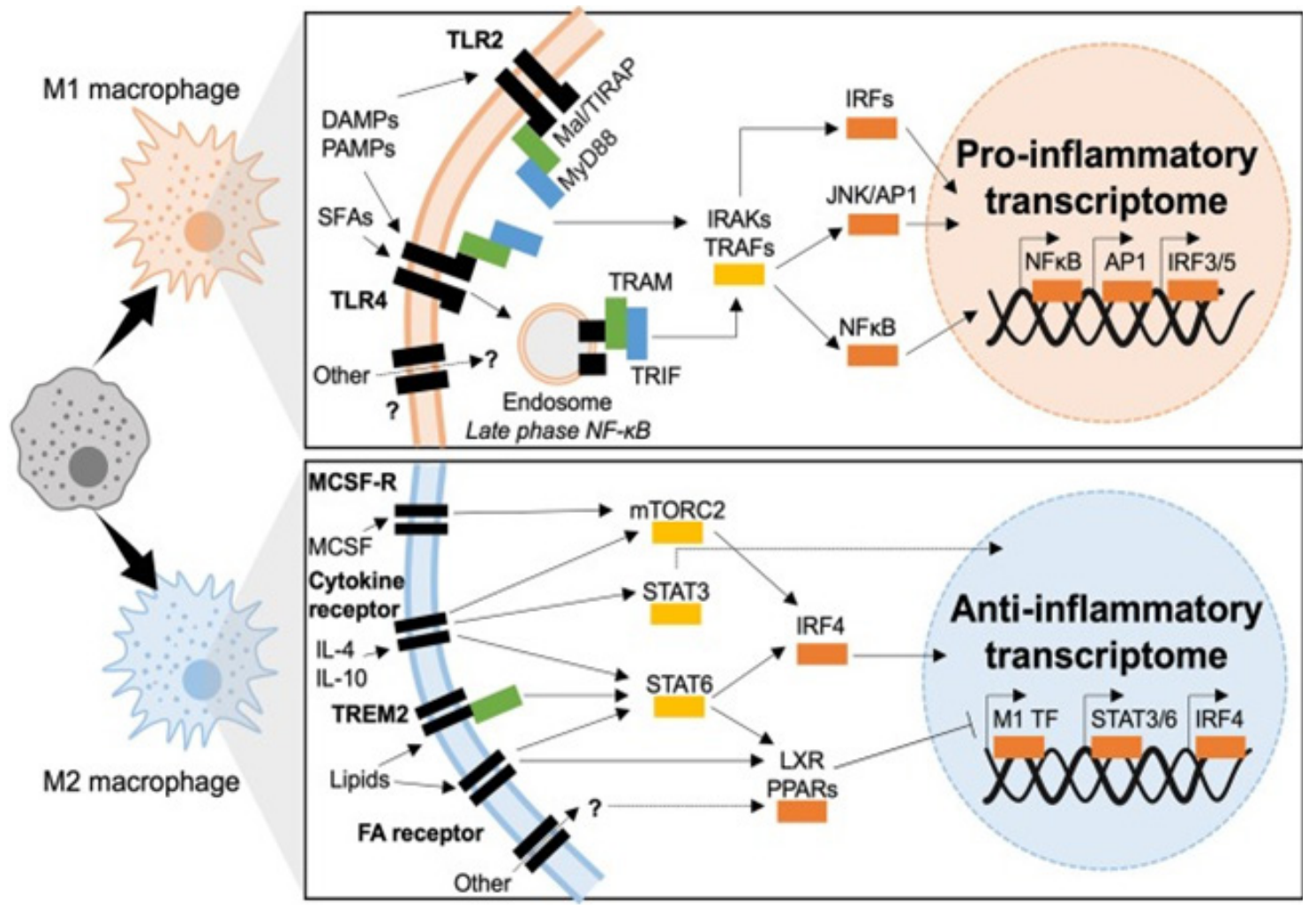

Figure 6 Transcriptional mechanisms of macrophage polarization in T2D. MI macrophages polarize in response to TLR2 or TLR4 ligation, downstream signaling is dependent of two adaptor complexes: Mal/TIRAP-MyD88 (TLR2 and TLR4) and TRAM-TRIF (TLR4). IRAK and TRAF signaling then dictate which transcriptional programmes are engaged, IRF3 IRF5, API, and NFKB have been shown to induce inflammatory polarization in T2D. M2 macrophages polarize downstream of cytokine and fatty acid receptor stimulation (also possible through other molecules such as TREM2). Intermediate signaling is largely through STATs and nuclear receptors (LXR and PPAR). STATs act as transcription factors or activate IRF4, whereas nuclear receptors act through transrepression of MI transcription factors (MI TF). ${ }^{189}$

\section{Inflammation in T2D-TLR-dependent}

Highly conserved transmembrane receptors namely TLR's are expressed both in along with on macrophages. This is secondary to the evolutionary need for recognition of structurally conserved molecules as well as pathogens. ${ }^{130}$ Every TLR from TLR 1 to TLR3 can recall specific ligands varying from LPS, to nucleic acids, viral particles as well as chitin. Besides their canonical part in host-defense, various TLR's are involved in metabolic inflammation as well as IR. ${ }^{131}$ That way TLR's can recognize besides infectious pathogens via PAMP's, metabolic stressors or DAMPs correlated with sterile inflammation as well as glucolipotoxicity. The basic TLR's involved with diabetogenesis are TLR 2 as well as TLR4. When these 2 TLR's get engaged chronic inflammation as well as IR results via direct 
interference with insulin signaling. ${ }^{132}$ TLR 2 as well as TLR4 shares common adaptor proteins in macrophages, the myeloid differentiation primary response (MyD 88) protein as well as Mal/TIRAP which recruit IRAK kinases on TLR binding as well as dimerization. IRAK 1,2 as well as 4 downstream signaling activates NFKB as well as activator protein 1(AP1). Further TLR4 activates other downstream signaling as well. It represents the only TLR which forms complexes with all adaptor proteins, Mal/TIRAP as well as MyD 88 for initiation of early phase $\mathrm{NF \kappa B}$ responses, the complex then gets endocytosed along with endosomal TLR's correlate with TRAM as well as TRIF adaptors. Canonically TRAM as well as TRIF mobilizes the type1 interferon response that gets transcriptionally modulated via Interferon regulatory factors (IRF's). AP1 as well as late phase NFאB activation. Both early as well as late phase action is needed for manteinance of synthesis of inflammatory cytokines. ${ }^{132}$ Coordination of actions of TLR's, adaptor proteins as well as kinases causes sustained activation of 3 main transcriptional programmes, led by IRF's, AP1, NFKB as well as JAK-STAT.

\section{Interferon regulatory factors (IRF's)}

In the beginning they were discovered for binding to virus inducible enhancer elements on Interferon coding areas, IRF's are well known for their regulation ability over innate immunity as well as type1 Interferon signaling. Further forming part of JAK-STAT signaling, IRF's give a response to multiple DAMP as well as PAMP's, modulate sterile inflammation(metabolic as well as autoimmune) as well as are active in non-immune cells as well (like adipocytes). ${ }^{133}$ Being 300-500 amino acids in length, IRF family members share a conserved $\mathrm{N}$ terminal DNA binding domain that helps in binding to Interferon sensitive regulatory elements .The $\mathrm{C}$ terminal IRF relation domain can be different and lets dimerization among various IRF's. ${ }^{134}$ IRF's1-5 along with IRF-9 regulate macrophage differentiation as well as polarization in response to PRR ligands, IRF's 3,4 as well as 5 have been documented to have a part in macrophage inflammation. ${ }^{133}$ IRF5 is responsible for macrophage polarization, along with having part in sterile inflammation besides autoimmunity like rheumatoid arthritis in which risk variants result in overexpression of IRF5 have been documented. ${ }^{133}$ In T2D, IRF5, aids in macrophage activation as well as macrophage failure in both AT as well as liver. IRF's are markedly expressed in ATM's by CD $11 \mathrm{c}+$ macrophages at CLS IRF5 expression as well as CLS development is markedly associated with AT inflammation, maladaptive adipocyte expansion in both local as well as systemic IR. ${ }^{77}$ On diet induced obese (DIO), mice possessing myeloid deficiency of IRF5 remain insulin sensitive inspite of escalated adiposity. Visceral white adipose tissue (WAT) in IRF5deficiency has property of adaptive remodeling that is modulated by a de facto type 2 immune response, that limits the adipocyte expansion as well as avoids losing sensitivity of insulin's antilipolytic action. ${ }^{77}$ Impaired expression of IRF5 is implicated in propagation of NASH. Right through NAFLD, IRF5 modulates proapoptotic as well as inflammatory signaling from liver macrophages towards lipotoxic hepatocytes. Continuous inflammatory signaling as well as hepatocyte apoptosis end in scarring fibrogenesis within liver. ${ }^{85}$ Canonically downstream of TLR4, IRF5 represents the active transcription factor. Notably, though the phenotypes of TLR4 deficiency as well as IRF5 deficiency are practically similar under DIO, this TLR4- IRF5 axis needs to be corroborated experimentally in the etiopathogenesis of T2D as well as its complications. ${ }^{135}$ On the same line downstream of TLR4, IRF3 aids in AT inflammation on DIO as well as it inhibits AT browning. IRF3 deficient mice retain insulin sensitivity on high fat diet (HFD) as well as escalate AT browning. ${ }^{136}$ Converse to IRF3 as well as, IRF5, IRF4 aids in macrophage M2 Polarization as well as resolving inflammation. ${ }^{137}$ In IRF4 deficient mice, metabolic phenotype on HFD is thus exaggerated. ${ }^{138}$ Myeloid deficiency of IRF4 causes escalation of IR as well as AT inflammation as compared to IRF4 competent mice. ${ }^{138}$ Notably IRF4 gets nutritionally controlled via insulin signaling as well as by canonical transcription factors implicated in metabolic signaling (like FOXO1). Moreover IRF4 controls lipid handling within adipocyte, aiding in lipolysis by aiding in lipase expression.

\section{Activator protein I (API)}

AP1 represents a complex formed via proto-oncogenes c-Jun as well as c-Fos which is necessary for DNA binding. This activation of AP1 responds to cytokine signaling as well as growth factors; regulators apoptosis, cell growth as well as macrophage terminal differentiation to an M1 like phenotype. ${ }^{139}$ Action of AP1 gets regulated via post translational changes, mainly translocation, as well as dimerization of its subunits via signaling through c-Jun $\mathrm{N}$ terminal (JNK) as well as mitogen-activated protein kinases (MAPK). Action of AP1 further gets controlled via composition of its DNA binding dimer (Jun/ Jun, Jun/ Fos, b ZIP) as well as via binding partners. ${ }^{140}$ Canonically AP1 activation occurs in response to PRR ligation, cytokine signaling as well as growth factors. Regarding metabolic inflammation AP1 responds to saturated FA's (SFA) like palmitate. ${ }^{132}$ Macrophages that get exposed to palmitate liberate proinflammatory mediators in an AP1-based manner. ${ }^{132}$ Further Action of AP1 responds to hormone signaling, where leptin escalates a binding of nuclear proteins to the AP1 consensus sequence of the lipoprotein lipase (LPL) gene promoter. This action escalates macrophage expression of LPL, giving understanding into the mode of AP1 in foam cell development, atherogenesis as well as T2D. ${ }^{141}$ The upstream kinases which cause activation of AP1 subunits have been deeply evaluated in metabolic diseases like JNK'S. Mice deficient in JNK1 and/ or JNK2 remain metabolically healthy upon DIO, they gain lesser weight, are protected from IR as well as inflammation. ${ }^{142}$ Notably myeloid specific deficiency of JNK, causes non inflammatory obesity as well as reduction of serum FA's. Research points that myeloid- AP1 is a crucial modulator of AT lipolysis upon DIO. ${ }^{143}$

\section{Nuclear factor-kappa B (NFKB)}

$\mathrm{NF \kappa B}$ represents a transcription factor which facilitates M1 polarization, with it responding to multiple signals like cytokines, redox stress, bacterial or viral antigens as well as oxidized lipid. ${ }^{144,145}$ Impaired NFkB signaling takes place in a lot of inflammatory situations that include T2D. NFאB is markedly conserved in ATM's upon their M1/MMe differentiation and right through the onset of IR. Moreover cytokines liberated by M1/MMe Macrophages develop an amplifying loop which recruit as well as polarizes other leukocytes at the site of inflammation. Mice having myeloid deficiency of Inhibitor of NFKB kinase (IKK- $\beta$ ), NFKB's canonical activator protein, show a reduced inflammatory response by DIO as well as maintain systemic insulin sensitivity. ${ }^{146}$ Notably, hepatic deficiency of IKK- $\beta$ only retains insulin sensitivity in the liver (not in muscle, nor AT), pointing that the myeloid derived IKK- $\beta$ / NFKB is the main controller of systemic metabolic homeostasis. ${ }^{146}$

\section{Signal transducers and activators of transcription (STATs)}

STATs represent a family of transcription factors which control Interferon signaling, as well as have well known part in apoptosis; proliferation along with differentiation of innate immune cells. Importantly STAT activity is especially significant in sustaining 
immune tolerance. STAT's get activated downstream of cytokine, chemokine as well as growth factors signaling. STAT dimerization as well as nuclear translocation is based on phosphorylation modulated Janus kinase (JAK), that together form JAK-STAT pathway. STAT1 as well as 5 facilitate M1 like signaling while STAT 3 as well as 6 facilitates M2 like signaling in macrophages. ${ }^{147,148}$ Noticeably the more recent description of Mme phenotype is polarized independently of STAT1 activity. ${ }^{149}$ STAT1 in macrophages gets activated in response to high glucose and causes proinflammatory signaling via epigenetic modes. Notably glucose- responsiveness of STAT1 has been documented in vitro as well as ex vivo modeling, with little or no proof getting documented in vivo or via human studies on obesity, IR as well as T2D. ${ }^{150}$ Till date no proof links STAT 5 activation to diabetic pathogenesis inspite of its part in inflammatory polarization. STAT 3 have a powerful link to T2D formation as well as its sequelae, mainly with anti-inflammatory, metabolically protective characteristics. Like STAT 3 represents a downstream target of the 1st line therapy of T2D, metformin. Metformin inhibits differentiation of monocytes to macrophages as well as reduces their infiltration into atherosclerotic plaques via AMPK modulated inhibition of STAT 3. ${ }^{151}$ In same way IR as well as DIO, protection conferred by ABCA1/APOA1 action are STAT 3-based, like the anti-inflammatory AT phenotype of mice with a myeloid deficiency of JAK2. ${ }^{152}$ STAT 6 by itself or along with vasodilator stimulated phosphorylation (VASP) has immunoregulatory characteristics regarding metabolic inflammation. The (VASP-STAT axis has been explained in mice with a myeloid specific deficiency of VASP, mice had proneness towards inflammation of liver with IR in a STAT1 6 based manner. ${ }^{153}$ While STAT 6 deficiency predisposes mice to DIO, oxidative stress as well as AT inflammation. ${ }^{154}$

\section{Peroxisome proliferator activated receptor (PPAR)}

PPAR $\alpha, y$ as well as $\delta / \beta$, get expressed at various levels in variety of tissues and differentiation across stages of formation. Greatest expression levels are in live, skeletal along with cardiac muscle, spleen. They are involved in cellular metabolism, differentiation, development as well as control inflammation. In case of M1 macrophages PPAR $\alpha$ inhibits the expression of proinflammatory mediators via negative control of AP1 along with NFKB. various studies documented the advantage of PPAR $\alpha$ activation in T2D as well as its sequelae. PPAR $\alpha$ agonists have been used in T2D patients along with being effective in atherosclerosis, via inhibition of foam cell development as well as inflammatory signaling. This advantage is obtained via interference with c-Jun along with c-Fos interactions as well as by limiting lipid collection via repression of FA transport protein (FATP)-1. ${ }^{155,156}$ PPAR $\delta / \beta$ also acts on macrophage metabolism, in relation to efflux of lipids, of FA catabolism as well as $\beta$ oxidation. Activation of PPAR $\delta / \beta$ in macrophage controls whole body energy dissipation as well as systemic responses to cholesterol. Further activation of PPAR $\delta / \beta$ occurs secondary to dyslipidemia. ${ }^{157}$ In etiopathogenesis of T2D, PPAR $\delta / \beta$ activation has a protective part in regulating macrophages infiltration in AT as well as liver and aiding immune tolerance (M 2 Polarization) in ATM's acting downstream of STATs $6 .{ }^{158}$ Mice possessing myeloid deficiency of PPAR $\delta / \beta$ show an exaggerated metabolic phenotype upon DIO. PPAR $y$ has a significant part in adipose physiology, adipocyte differentiation as well as maturation. Of the 2 known isoforms, PPAR $y 1$ gets expressed within macrophages as well as adipocytes while PPAR y 2 gets expressed in adipocytes. ${ }^{159}$ PPAR y 1 escalates monocyte differentiation into M 2 macrophages besides being an inhibitor of inflammatory polarization, repressing matrix metalloproteinase $9, \mathrm{IL}-6, \mathrm{TNF}-\alpha$ as well as $1 \mathrm{~L}-1 \beta$ expression. ${ }^{155,160}$ In in vitro as well as ex vivo modeling, PPAR y inhibits M1 signaling related to LPS +IFNy stimulation, that includes iNOS cyclooxygenase 2 (COX-2), as well as a 1L- $12 .{ }^{161-163}$ Significantly macrophages, PPAR $y$ is also downstream target of internalized lipids, along with modulates expression of scavenger receptors ${ }^{164}$ for foam cell development. ${ }^{57}$ Hence PPAR $y$ deficient mice show impaired $\mathrm{M}$ 2 maturation as well as develop accelerated IR as well as metabolic inflammation in DIO. ${ }^{165}$ Notably, overexpression of PPAR $y$ show that mature adipocyte PPAR $y$ in fact is the major insulin sensitizing part(overexpression phenotype is comparable to TZD therapy. ${ }^{166}$ Little or nil benefit is seen in DIO if PPAR $y$ overexpression is there in macrophages. ${ }^{166}$ These over/under-expression studies show diverging functions of PPAR $y$. More work is needed in mode to find exact role as well as control of nuclear receptors as well as different isoforms in separate cell kinds as well as microenvironment. Multiple modes of action have been posited, with main being transrepression whereby PPAR $y$ binds to active proinflammatory transcription factors as well as represses their function. Repressive action via interaction with nuclear receptor corepressor (NCoR) complexes has also been hypothesized. ${ }^{167}$

\section{Liver X receptor's (LXR's)}

LXR's are present in 2 isoforms LXR $\alpha$ as well as LXR $\beta$, both of which are lipid activated as well as control macrophage inflammatory responses. For regulation of transcription LXR's heterodimerize with retinoid X Receptor (RXR) as well as bind to LXR response elements on the genome. ${ }^{168}$ LXR's have a significant part in T2D besides cardiovascular disease (CVD) promoting antiinflammatory polarization as well as controlling macrophage lipid amount. Activaton of LXR's by oxysterol species along with synthetic compounds lets cholesterol efflux via macrophages besides lipid transporters ACBCA1 as well as ABG. ${ }^{169}$ LXR's further directly repress transcription of proinflammatory genes as well as escalate transcription of anti-inflammatory genes in response to polarizing stimuli. ${ }^{170}$ LXR's because their action by transrepression once they get simulated. This alteration avoids LPS-based exchange of corepressor, hence sustaining LXR's-modulated expression of inflammatory transcription factor activity. ${ }^{171}$ Various reports demonstrate the protective roles that LXR's have in metabolic-inflammation as well as IR. LXR's agonists act as insulin sensitizers as well as controllers of glycaemia via repression of hepatic gluconeogenesis. ${ }^{172}$

\section{Hypoxia inducible factor I (HIF I)}

HIF 1 represents a transcription factors having 2 subunits, $\alpha$ as well as $\beta$. HIF $1-\alpha$ gets stabilized with its expression escalated in response to Hypoxia, while HIF 1- $\beta$ gets constitutively expressed as well as stabilized independent of $\mathrm{O} 2$ amounts. ${ }^{161}$ Under hypoxic situations HIF $1-\alpha$ translocate to nucleus as well as dimerizes with HIF $1-\beta$ that lets the binding of hypoxia response elements (HREs) on the genome along with controlling target gene expression. ${ }^{162}$ In $\mathrm{O} 2$ poor situations HIF 1 activation causes a shift towards anaerobic respiration in cells in which bioenergetic needs get supported via glucose metabolism. ${ }^{163}$ Myeloid specific overexpression of HIF 1- $\alpha$ specific removal of HIF 1- $\alpha$ impairs murine models of obesity, modes of M1 polarization in ATM's are only partially dependent on HIF 1- $\alpha$ activation. Myeloid specific removal of HIF $1-\alpha$ cause reduced inflammatory signaling, reduced CLS development as well as abrogated metabolic phenotype upon DIO. ${ }^{173}$

\section{Macrophage polarization-epigenetic regulation}

Epigenetic mechanisms regulate chromatin structure as well as conformation, factors that determine the accessibility of genetic loci to transcription factors. Epigenetic remodeling, via transcriptional coregulator as well as epigenetic modifying enzymes (like histone 
deacetylases or HDACs), control transcription factor action. Getting insight into epigenomic regulatory mechanisms can aid in formation of newer treatments, like blocking an unwanted pathway or reprogramming macrophages to get a phenotype that is of greater advantage. Fast stimulation of an inflammatory transcriptional profile is a classic property of macrophage activation needed for an immune response that works. In steady state coregulator complexes bind to genomic areas of a wide repertoire of inflammatory genes for sustenance of macrophages in a quiescent status; this mode prevents deregulated inflammatory gene stimulation. Coregulator act by $1^{\text {st }}$ recalling transcription factor activity and subsequently they further manipulate this activity via generation of crosstalk with the transcriptional mode along with chromatin. ${ }^{174}$ Coregulator can be classified as coactivator or corepressor. Coactivator recognizes as well as stimulates active transcription, while corepressor recognizes and represses inactive transcription. But this classification of coregulator activity does not properly reflect the physiological or physiopathological condition as coregulator activator or repressive function is totally dependent on context. Coregulator develop cell type dependent as well as ligand dependent epigenomes by developing large multiprotein complexes that "write' 'erase " or "read " reversible chromatin modifications related to transcriptional Activity Figure 6, although underlying mode is still not clear .Lot of explanations that give conviction says changes in function or expression of coregulator is central to the impairment of transcription that is inherent to disease particular epigenomes. 2 such corepressor are the nuclear receptor corepressor ( $\mathrm{NCoR} 1)$ as well as silencing mediator of retinoic acid as well as thyroid hormone receptor (SMRT or NCoR2), which cross react with inflammatory transcription factor like AP1 as well as a NFKB and in turn bind to particular genomic regions to control transcription. ${ }^{174}$ The general opinion was that on TLR4 stimulation the NCoR complex gets liberated via promoter /enhancer areas of inflammatory gene for promoting or depressing, their transcription. ${ }^{175}$ But in many cases this separation does not actually point the in vivo condition, with context-related microenvironment signals stimulation coregulator properties. Regarding macrophage polarization as well as T2D, particular removal of $\mathrm{NCoR}$ in macrophages result in transcriptional activation of LXR, ending in lipogenic enzymes getting induced ,that further result in local anti-inflammatory actions via repression of NFkB. ${ }^{162} \mathrm{NCoR}$ causes pro inflammatory actions in macrophages. As with NCoR, macrophages via HDAC3-deficient phenotypes were independent of anti-inflammatory as seen in 2 independent studies that came as a surprise. ${ }^{176}$ These observations were separate from earlier work demonstrating that HDAC3 as well as NCoR were demonstrated to assemble a repressive complex through crosstalk with the NFKB subunit $\mathrm{p} 50$, essential for the TLR tolerance process in which they maintained TLR 4 activation represses inflammatory gene expression. ${ }^{174}$ Greater details are needed for getting a good insight into the particular mode of action of HDAC3 as well as NCoR.

Conversely anti-inflammatory actions have been thought to be due to the SMRT/GPS2 (G-protein pathway suppressor 2) subunit complex. In their recent study in 2016 Fan et al., ${ }^{177}$ showed that macrophage specific knockout of the GPS2 subunit accelerates metabolic inflammation, escalating glucose homeostasis under metabolic stress. ${ }^{177}$ This phenotype gets correlated with genomic features of the GPS2 repressive pathway, that implicates direct repression of the c-Jun subunit of AP1. Noting all the current studies, 2 sub complexes have separate functions; SMRT/GPS2 might have anti-inflammatory actions while $\mathrm{HDAC} 3$ as well as $\mathrm{NCoR}$ might act as pro inflammatory machinery. This might answer the contradiction in phenotypes of the respective $\mathrm{KO}$ models, inspite both getting classified to start with as corepressor complexes. Sub complex specifications would help in regulating transcription of separate gene clusters in response to different signals and probably end in separate interactions with TF's, coregulator as well as chromatin parts like histones. This gets exemplified via GPS2 acting on other cell kinds. Actually the anti-inflammatory actions of GPS2 get conserved in adipocytes by repression of CCL2 as well as IL- $6 .{ }^{178}$ Whereas the major part of GPS2 in hepatocytes is of repression of the metabolic nuclear receptor PPAR alpha action. ${ }^{179}$ Hepatocyte specific knockout of GPS2 is then protective on inflammatory stimulus while adipocyte or macrophage particular GPS2-deficiency is harmful for whole body glucose homeostasis and show accelerated inflammation. These reverse functions are also seen in humans. ${ }^{177,179}$ These associations have significance since they suggest the probability that inexact GPS2 action might be associated with macrophage pathways which drive AT dysfunction as well as IR. Other coregulator, like glutamate receptor-interacting protein (GRIP-1) control macrophage programmed responses to IL-4 via action as a coactivator for Kruppellike factor (KLF-4), that is known to drive tissue resident macrophage differentiation. ${ }^{180}$ Obese mice having conditional macrophage specific removal of GRIP-1 form inflammation and followed by macrophage infiltration in metabolic tissues, fatty livers, hyperglycemia as well as IR, that recapitulates metabolic disease via GRIP-1's glutamate receptor independent actions. Hence coregulator like GPS2, GRIP1 , NCoR as well as HDAC3 is key controllers of macrophage reprogramming in metabolic disease. Their coordinated actions involve transcriptional modes which coordinate a balance among macrophage polarization states as well as subpopulations for manteinance of metabolic homeostasis Figure 7. Epigenetic remodeling of particular histones also represents an indicator of macrophage activation states. Macrophage Activation can get controlled by trimethylation of lysine 27 on histone 3 (a modification known as H3K27me3) through the action of lysine specific demethylase 68(KDM6B; also called JMJD3). The histone mark H3K27me3 represses transcription and is deposited via histone lysine $\mathrm{N}$ methyl transferase $(\mathrm{EZH}-2$ a subunit of the Polycomb Repressive Complex 2.While removing histone mark gets modulated via H3K27me3 demethylase KDM6A as well as KDM6B. The crucial part of EZH-2 histone methyl transferase modification was documented by Zhang et al., ${ }^{181}$ in changing of macrophage phenotype. ${ }^{181}$ EZH-2 regulates H3K27me3 deposition on the promoter of SOCS3, which encodes a cytokine signaling repressor .Thus mice having a myeloid specific deficiency of EZH-2 show attenuation of macrophage activation as well as decreased inflammatory under models of autoimmune disease. These findings make EZH-2 at luring target for other inflammatory diseases like T2D.

The part of KDM6B in macrophage is not known. Excellent studies posited that KDM6B is not essential for the polarization of the pro inflammatory macrophages phenotype in mice but is needed for an appropriate anti-inflammatory response through the removal of H3K27me3 from the IRF4 promoter. ${ }^{182}$ Absence of KDM6B totally blocks the macrophage induction in mice that have been challenged via helminthes or chitin, pointing a part of KDM6B must be more in M2 as compared to M1 macrophages. ${ }^{183}$ Conversely pro inflammatory TLR4 gene activation was reduced in KDM6B-deficient macrophages. Thus targeting KDM6B H3K27me3 demethylases with small molecule inhibitors impairs inflammatory responses in human primary macrophages and hence might be of lot of pharmacological significance for treating inflammatory diseases that includes T2D. ${ }^{184,185}$ Notably KDM6B further manipulates expression of chemokines dependent on granulocyte macrophages colony stimulating factor (GMCSF) stimulation, that normally works via STAT5-modulated as well as IRF5 modulated induction of a pro inflammatory phenotype. Epigenetic signatures vary in disease state of chronic inflammation, 
like in T2D. KDM6B is one of the little Epigenetic modifiers which might have a direct involvement in changing the epigenetic signatures of macrophages. Role of KDM6B in regulating macrophages expression of IL-12 in a DM context was 1st documented by Gallagher et al., ${ }^{184}$ In a recent study macrophages that got treated with a selective KDM6B inhibitor demonstrated change in expression of pro inflammatory cytokines that gave proof for Gallagher's study. ${ }^{184}$ Still it is to be found that the degrees of contribution of nutrient over intake as well as obesity, IR or hyperglycemia ${ }^{185}$ to changes seen in histone methylation. A recent study posits that changed DNA methylation is mainly a result of adiposity, instead of a cause. ${ }^{186}$

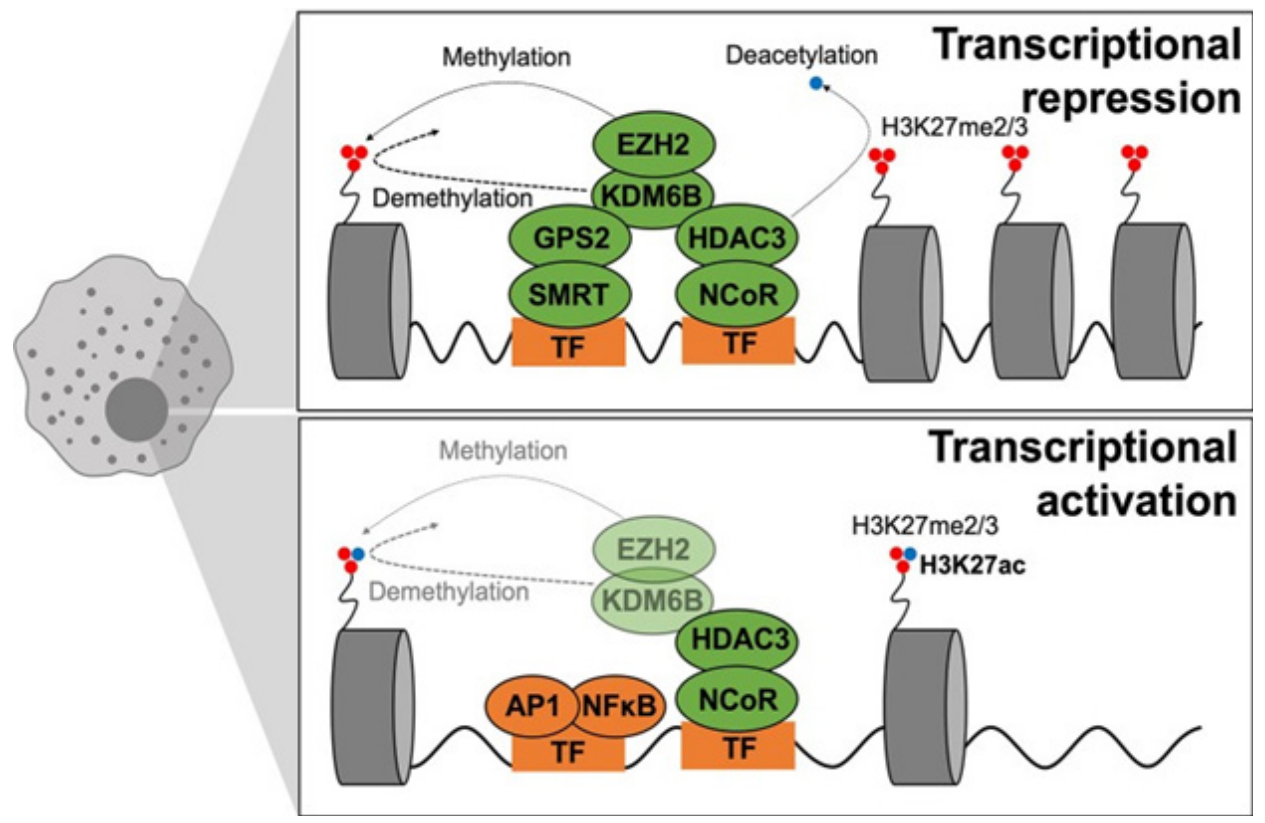

Figure 7 Epigenetics of macrophage polarization in T2D. Epigenetic mechanisms that modulate transcription act on chromatin remodeling and altering DNA accessibility to transcriptional machinery. Modifications are in dynamic exchange where methylation and acetylation status of $\mathrm{H} 3 \mathrm{~K} 27$ dictate repression or activation of transcription, respectively. When chromatin is closed, methylation is dynamically altered by EZH2 (adding methyl groups) and KDMB6 (removing methyl groups), and the active transcription mark (H3K27ac) is removed through HDAC3 activity. GPS2-SMRT also participates in gene repression. During active transcription, the active transcription mark (H3K27ac) is maintained whilst EZH2-KDM6B play smaller roles. GPS2-SMRT is not present to exert repressive effects. ${ }^{189}$

\section{Conclusion}

Lot of advances has been made in last decades that implicated tissue macrophages in IR formation. Actually macrophages have been found to be the central players in sustaining tissue as well as organism homeostasis in response to every day challenges of temporary over as well as under nutrition-from inflammatory signaling essential for insulin liberation, to the house keeping part they have in buffering AT lipolysis as well as non inflammatory signaling in NAFLD. Till date concentration of studies had been on getting to know the molecular modes which regulate macrophage responses to dysmetabolism, basically restricting classification into M1 like vs M2 like macrophages. With the recent technological improvement of single cell sequencing have given us to give a much >in depth classifying macrophage subsets, i.e. immune cell ontogeny, hence single cell sequencing has resulted in a functional reclassification of innate immune subtypes. ${ }^{16}$ Significance of these studies lies in giving the properties of macrophages in tissue niches which had been overlooked till now, like pancreatic islet macrophages or SNS related macrophages. This switch in paradigm in macrophage functional reclassification can further be extended to their metabolic properties, their bioenergetic needs as well as adaptations to the particular challenges of IR. Various studies of infection as well as immunity have mostly used typical immune cell activation. Tissue macrophage bioenergetics needs to be found at the formation stage, at steady state as well as at onset of IR. Macrophage metabolism is a luring therapeutic target which will manipulate inflammation without markedly influencing effector functions by turning the immune response " on" or "off". After recent findings of non immune as well as non inflammatory signaling via macrophages, we have gained insight into noncanonical part of the innate immune cells, like dendritic cells, NK cells, as well as ILC's. Since innate immunity, with all its diverseness, we know maintains homeostasis, without definitely engaging inflammation, steady state characteristics as well as responses to physiological variation need to be mapped to get more understanding of deregulation of innate immune effector function that causes metabolic pathology. Inspite of consistent strong correlations as well as modes relation between inflammation as well as IR there have been relatively little successful translation innovations. Antidiabetic therapy at present aims to normalize glycaemia via a variety of modes and have been demonstrated to also buffer systemic inflammation (like TZDs, DPP4 inhibitors, GLP-1, RAs). These positive effects implicate improvement in the inflammatory profile for improvement of metabolic responses. ${ }^{188}$ Knowing that lot of proof is there that macrophage polarization is central to T2D pathology very few clinical trials target inflammation in T2D.

Till date anti- inflammatory strategies in clinical trials have targeted cytokines with neutralizing antibodies (like ant-TNF. Anti IL1) or have applied agents that have uncharacterized modes (like chloroquine, diacrein). Studies on these drugs have been promising; enhance insulin sensitivity secretion or fasting blood glucose secretion. ${ }^{189,190}$ The biggest problems in their routine use are absence of long-term studies 
.Other problems in translation of anti- inflammatory methods is the fact that inflammation in T2D has multiple causes, with the disease itself predisposing patients to a group of complicated sequelae as well as comorbidities (in a case where the increase of precision medicine tries to find modes of response or those at risk). Technical barriers further effect translational potential like clinical trials investigating inflammation on the basis of relatively nonspecific circulating markers like CRP, that at best reflects systemic inflammation. While in preclinical studies scientists aim to examine tissue specific inflammation, extrapolating these to human studies is a big hurdle. Specifically delivering drugs to macrophages also Is a big challenge clinically as well as leaves the door open for unanticipated side effects .With the above work promising methods are slowly enhancing the translation potential of targeting inflammation in Metabolic diseases, like repurposing of well tolerated agents from other pathologies or fields, as was the case with anti-malarial chloroquine as well as hydroxyl chloroquine as well as diacrein used for treatment of arthritis. In basic research, >attention is being given in earlier disease course, where mechanisms which might delay or negate the natural course of T2DM are getting described and will give the basis for innovative therapeutic targets. Developing small molecule inhibitors or antisense oligonucleotides are luring on targeting epigenetic or transcriptional pathways and are proving to be of $>$ value in clinical research community. In same line search for metabolic immunogens or characterization of circulating immune cell populations will aid in forming predictive biomarkers of susceptibility to diseases or at risk proneness of disease propagation once IR has got established.

\section{Acknowledgments}

None.

\section{Conflicts of interest}

The authors declare that there are no conflicts of interest.

\section{Funding}

None.

\section{References}

1. Shimobayashi M, Albert V, Woelnerhanssen B, et al. Insulin resistance causes inflammation in adipose tissue. J Clin Invest. 2018;128(4):15381550 .

2. Johnson AM, Okefsky JM. The origins and drivers of insulin resistance. Cell. 2013;152(4):673-684.

3. Wu H, Ballantyne CM. Skeletal muscle inflammation and insulin resistance in obesity. J Clin Invest. 2017;127(1):43-54.

4. Rosso M, Kimbrough DJ, Gonzalez CT, et al. Cross-sectional study of smoking exposure: no differential effect on OCT metrics in a cohort of MS patients. Mult Scler J Exp Transl Ther. 2019;5(1):2055217319828400.

5. Jager J, Aparicio-Vergara M, Aouadi M. Liver innate immune cells and insulin resistance: the multiple facets of Kupffer cells. Int Med. 2016;280(2):209-220.

6. Kochar KK, Allahbadia G, Singh M. An update on a etiopathogenesis and management of obesity. Obes Control Ther. 2016;3(1):1-17.

7. Kochar KK, Allahbadia GN, Singh M. Current management of obesity in an infertile female. Recent advances and future prospectives. Drugs $J$ Pharm Nutr Soc. 2013;3:1-13.

8. Kochar KK, Allahabadia GN, Singh M. Further update on the management of obesity with emphasis on genetic perspective. BAOJ Obe Weigh Manage. 2016;3(1):1-17.
9. Kochar KK, Allahabadia GN, Singh M. A review on nutrient metabolism with special emphasis on fatty acid metabolism. BAOJ Food Sci \& Tec. 2017;1(1):001.

10. Kulvinder KK, Allahbadia GN, Singh M. Hypothalamic inflammation and glioses as aetiopathogenetic factor inhigh fat diet induced obesity and various therapeutic options to resolve it. Obes Res Open J. 2017; 4(2): 44-60.

11. Kulvinder KK, Allahbadia GN, Singh M. Current advances in pathogenesis in obesity: role of hypothaalamic glioses. J Obes Weight Loss. 2018;3(008):1-11

12. Kulvinder KK, Allahbadia GN, Singh M. Weight loss associated with high protein intake in obesity: interactions of gut microbiota in protein sources influencing this positive effect. Acta Scientific Nutritional Health 2018;2(7):80-89.

13. Kulvinder KK, Allahbadia GN, Singh M. Impact of nutrigenomics on various metabolic disorders in relation to life style alteration. Austin J Nutri Food Sci. 2018;6(1):1100.

14. Kulvinder KK, Allahbadia GN, Singh M. Synthesis and functional significance of poly unsaturated fatty acids (PUFA's) in body. Acta Scientific Nutritional Health. 2018;2(4);43-50.

15. Kulvinder KK, Allahbadia GN, Singh M. Will utilization of resveratrol's effects be practical in multiple chronic inflammatory diseases and autoimmune diseases: a detailed review of its immune responses an further clinical development in humans in future-a systematic review. Acta Scientific Microbiology. 2019:14-23.

16. Kulvinder Kochar Kaur, Allahbadia GN, Singh M. A comprehensive review explaining the detailed mechanism of actions of various lentils Like soyabeans, chickpeas in improving insulin resistance. Acta Scientific Nutritional Health. 2019;3(4):53-65.

17. Kulvinder KK, Allahbadia GN Singh M. Alteration in natural killer (NK) cell Function in obesity-correlating with comorbidities development like cancer and type 2diabetes-a minireview. J Endocrinol. 2019:3(2):000140

18. Kulvinder KK, Allahbadia GN, Singh M. Importance of controlling obesity and associated immune response changes in prevention and treatment of cancer-with plant therapies of help. Adv Obes Weight Manag Control. 2019;9(4):114-116.

19. Kulvinder KK, Allahbadia GN, Singh M. Monoterpenes-a class of terpenoid group of natural products as a source of natural antidiabetic agents in the future-a review. CPQ Nutrition. 2019;3(4):1-21.

20. Kulvinder KK, Allahbadia GN, Singh M. Bioactive compounds within herbs and spices contributing to anti diabetic action in type 2 diabetes mellitus (T2DM)-a short communication. Acta Scientific Nutritional Health. 2020;4(1):88-92.

21. Kulvinder KK, Allahbadia GN, Singh M. An update on bariatric surgery with long term efficacy and its utilization for medical therapy development from the different mechanism of action and other short comes to be outcome. BAOJ Surgery. 2018;4: 038.

22. Kulvinder KK, Allahbadia GN, Singh M. How do we apply advances in knowledge of hepatic macrophages in treating liver diseases especially non-alcoholic fatty liver disease (NAFLD), non-alcoholic steatohepapititis (NASH), with the increasing incidence of diabesity-a systematic review. EC Endocrinology and Metabolic Research. 2020.

23. Kulvinder Kochar Kaur, Allahbadia GN, Singh M. Will probiotics provide the answer for therapy of non-alcoholic fatty liver disease (NAFLD)?-a systematic review. Biochem Physiol. 2020;9:257.

24. Hotamisligil GS, Shargill NS, Spiegelman BM. Adipose expression of tumor necrosis factor alpha: direct role in obesity linked insulin resistance. Science. 1993;259(5091):87-91.

25. Weisberg SM, McCann D, Desai M, et al. Obesity is associated with macrophage accumulation in adipose tissue. $J$ Clin Invest. 2003;112(12):1796-1808. 
26. Jaitin DA, Adlung L, Thomas CA, et al. Lipid-associated macrophages control of metabolic homeostasis in a trem 2-dependent manner. Cell. 2019;178(3):686-698.e14.

27. Dong X, Liu J, Xu Y, et al. Role of macrophages in experimental liver injury and repair in mice. Exp Ther Med. 2019;17(5):3835-3847.

28. Dalmas F, Lehmann FM, Dror E, et al. Interleukin 33 activated isletresident innate lymphoid cells promote insulin secretion through myeloid cells retinoic production. Immunity. 2017;47(5):928-942.e7.

29. Perdiguero EG, Gersmann F. The development and maintainance of resident macrophages. Nat Immunol. 2016;17(1):2-8.

30. Stout RD, Jiang C, Matta B, et al. Macrophages sequentially change their functional phenotype in response to changes in microenvironmental influences. J Immunol. 2005; 175:342-49.

31. O'Neil LA, Kishton RJ, Rathmell J. A guide to immunometabolism for immunol ogists. Nat Rev Immunol. 2016;16(9):553-565.

32. Martinez FO, Gordon S. The M1 and M2 paradigm of macrophages activation: time for reassessment. F1000 Prime Rep. 2014;6:13.

33. Mantovani A, Sica A, Sozzani S, et al. The chemokine system in diverse forms of macrophages activation and polarization. Trends Immunol. 2004;25(12):677-686.

34. Ginhoux F, Schultze JL, Murray PJ, et al. New insights into the multidimensional concept of macrophage ontogeny, activation and function. Nat Immunol. 2016;17(1):34-40.

35. Amouzou C, Breuker C, Fabre O, et al. Skeletal muscle insulin resistance and absence of inflammation characterize insulin resistant grade I obese women. PLoS One. 2016;11(4):e0154119.

36. Bhatt M, Rudrapatna S, Banfield L, et al. Evaluating the evidence for macrophage presence in skeletal muscle and its relation to insulin resistance in obese mice and humans: a systematic review protocol. $B M C$ Res Notes. 2017;10(1):374.

37. Calderon B, Carrerro JA, Ferris ST, et al. The pancreas anatomy conditions the origin and properties of resident macrophages. J Exp Med. 2015;212(10):1497-1512.

38. Ehses JA, Perren A, Eppler E, et al. Increased number of islets associated macrophages in type 2 diabetes. Diabetes. 2007;56(9):2356-2370.

39. Carrerro JA, McCarthy DP, Ferris ST, et al. Resident macrophages of pancreatic islets have a seminal role in the initiation of autoimmune diabetes of NOD mice. Proc Natl Acad Sci USA. 2017;114(48):E10418E10427.

40. Zinselmyer BH, Vomund AN, Saunders BT, et al. The resident macrophages in murine pancreatic islets are constantly probing their local environment, capturing beta cell granules and blood particles. Diabetologia. 2018;61(6):1474-1483.

41. Weitz JR, Makhmutosva M, Almaca J, et al. Mouse pancreatic Islet macrophages use locally released ATP to monitor beta cell activity. Diabetologia. 2018;61(1):182-192.

42. Böni-Schneitzler M, Boller S, Debray S, et al. Free Fatty acids induce a proinflammatory response in islets via abundantly expressed interleukin 1 receptor. Endocrinology. 2009;150(12):5218- 5229.

43. Hajmrle C, Smith N, Spigelman AF, et al. Interleukin 1 signaling contributes to acute islets compensation. JCI Insight. 2016;1(4):e86055.

44. Burke SJ, Batdorf HM, Burk DH, et al. Pancreatic deletion of the interleukin-1 receptor disrupts whole body glucose homeostasis and promotes $\beta$-cell de-differentiation. Mol Metabol. 2018;14:95-107.

45. Dror E, Dalmas E, Meier DT, et al. Postprandial macrophage-derived, IL$1 \beta$ stimulates insulin and both synergistically promote glucose disposal and inflammation. Nat Immunol. 2017;18(3):283-292.
46. Chitterbath M, Gunaselan D, Zheng X, et al. Islets macrophages are associated with islet vascular remodeling and compensatory hyperinsulinemia during diabetes. Am J Physiol Endocrinol Metab. 2019;317(6):E1108-E1120.

47. Ying W, Lee YS, Dong Y, et al. Expansion of islet-resident macrophages leads to inflammation affecting beta cell proliferation and function in obesity. Cell Metab. 2019;29(2):457-474.

48. Eguchi K, Manabe I, Oishi-Tanaka Y, et al. Saturated fatty acid and TLR signaling link beta cell dysfunction and islet inflammation. Cell Metab. 2012;15(4):518-533.

49. Segerstolpe A, Palasantza A, Eliasson P, et al. Single cell transcriptomic profiling of human pancreatic islets in health and type 2 diabetes. Cell Metab. 2016;24(4):593-607.

50. Kane H, Lynch I. Innate immune control of adipose tissue homeostasis. Trends Immunol. 2019;40(9):857-872.

51. Fischer-Posovszky P, Wang QA, Asterholm IW, et al. Targeted deletion of adipocytes by apoptosis leads to adipose tissue recruitment of alternatively activated M2 macrophages. Endocrinology. 2011;152(8):3074-3081.

52. Kosteli A, Sugaru E, Haemmerle G, et al. Weight loss and lipolysis promote a dynamic immune response in murine adipose tissue. $J$ Clin Invest. 2010;120(10):3466-3479.

53. Nguyen KD, Qiu Y, Qiu X, et al. Alternatively activated macrophages produce catecholamines to sustain adaptive thermogenesis. Nature. 2011;480(7375):104-108.

54. Aaoudi M, Vangala P, Yawe JC, et al. Lipid storage by adipose tissue macrophages regulates glucose tolerance. Am $J$ Physiol Endocrinol Metab. 2014;307(4):E374-E383.

55. Xu X, Grijalva A, Skowronski A, et al. Obesity activates a program of lysosomal-dependent Lipid metabolism in adipose tissue macrophage independently of classic activation. Cell Metab. 2013;18(6):816-830.

56. Flaherty SE 3rd, Grijalva A, Xu X, et al. A lipase-independent pathway of lipid release and immune modulation by adipocytes. Science. 2019;363(6430):989-993.

57. Nagy I, Tontonoz P, Alvarez JG, et al. Oxidized LDL regulates macrophage gene expression through ligand activation of PPAR gamma. Cell. 1998;93(2):229-240.

58. Pirzgalska RM, Seixas E, Seidman JS, et al. Sympathetic neuronassociated macrophages contribute to obesity by importing and metabolizing norepinephrine. Nat Med. 2017;23(11):1309-1318.

59. Orr S, Kennedy A, Andersen-Baucum EK, et al. Obesity alters adipose tissue macrophage iron content and tissue iron distribution Diabetes. 2014;63(2):421-432.

60. Gabrielson JS, Gao Y, Simcox JA, et al. Adipocyte iron regulates adiponectin and insulin sensitivity. J Clin Invest. 2012;122(10):35293540 .

61. Bourlier V, Zakaroff-Girard A, Miranville A, et al. Remodeling phenotype of human subcutaneous adipose tissue macrophages. Circulation. 2008;117(6):806-815.

62. Lumeng CN, Bodzin JL, Saltiel AR. Obesity induces a phenotypic switch in adipose tissue macrophage polarization. J Clin Invest. 2007;117(1):175184

63. Kim HT, Higashimori T, Park SY, et al. Differential effects of interleukin-6 and -10 on Skeletal muscle and liver insulin action for in vivo. Diabetes. 2004;53(4):1060-1067.

64. Bluher M, Fasshauer M, Tonjes A, et al. Association of interleukin-6, Creactive protein, interleukin-10 and adiponectin plasma concentrations with measures of obesity, insulin sensitivity and glucose metabolism. Exp Clin Endocrinol Diabetes. 2005;113(9):534-537. 
65. Kowalski JM, Nicholls HT, Risis S, et al. Deficiency of haematopoieticcell derived IL-10 does not exacerbate high-fat-diet-induced inflammation or insulin resistance in mice. Diabetologia. 2011;54(4):888-899.

66. Ying W, Riopel M, Bandopadhyay G, et al. Adipose tissue macrophagederived exosomal miRNAs can modulate in vivo and in vitro insulin sensitivity. Cell. 2017;171(2):372-384.

67. Zheng C, Yang Q, Cao J, et al. Local proliferation initiates macrophage accumulation in adipose tissue during obesity. Cell Death Dis. 2016;7(3):e2167.

68. Amano SU, Cohen IL, Vangala P, et al. Local proliferation of macrophages contributes to obesity-associated adipose tissue inflammation. Cell Metab. 2014;19(1):162-171.

69. Ramkhelawon B, Hennessy EJ, Ménager M, et al. Netrin 1 promotes adipose tissue macrophages retention and insulin resistance in obesity. Nat Med. 2014;20(4):377-384.

70. Prieur X, Mok CY, Velagapudi VR, et al. Differential lipid partitioning between adipocytes and tissue macrophages modulates macrophages lipotoxicity and M2/M1 polarization in obese mice. Diabetes. 2011;60(3):797-809.

71. Hill DA, Lim HW, Kim YH, et al. Distinct macrophages populations direct inflammatory versus physiological changes in adipose tissue. Proc Natl Acad Sci USA. 2018;115(22):E5096-E5105.

72. Kratz M, Coats BR, Hisert KB, et al. Metabolic dysfunction drives a mechanically distinct proinflammatory phenotype in adipose tissue macrophages. Cell Metab. 2014;20(4):614-625.

73. Cinti S, Mitchell G, Barbatelli G, et al. Adipocyte death defines macrophages localization and function in adipose tissue of obese mice and humans. J Lipid Res. 2005;46(11):2347-2355.

74. McNally AK, Andersson JM. Macrophage fusion and multinucleated giant cells of inflammation. Adv Exp Med Biol. 2011;713:97-111.

75. Patsouris D, Li PP, Thapar D, et al. Ablation of CD 11cpositive cells normalizes insulin sensitivity in obese insulin resistant animals. Cell Metab. 2008;8(4):301-309.

76. Weiss M, Byrne AJ, Blazek K, et al. IRF5 controls both acute and chronic inflammation. Proc Natl Acad Sci USA. 2015;112(35):11001-11006.

77. Dalmas E, Toubal A, Alzaid F, et al. Irf5 deficiency in macrophages promotes beneficial adipose tissue expansion and insulin sensitivity during obesity. Nat Med. 2015;21(6):610-618.

78. Sindhu $\mathrm{S}$, Thomas $\mathrm{R}$, Kochumon $\mathrm{S}$, et al. Increased adipose tissue expression of Interferon regulatory factor 5(IRF5) in obesity: association with metabolic inflammation. Cells. 2019;8(11):1418.

79. Scott CL, Zheng F, De Baetselier P, et al. Bone marrow-derived monocytes give rise to self-renewing and fully differentiated kupffer cells. Nat Commun. 2016;7:10321.

80. Ju C, Tacke F. Hepatic macrophages in homeostasis and liver diseases: from pathogenesis to novel therapeutic strategies. Cell Mol Immunol. 2016;13(3):316-327.

81. Burt AD, Ferrell LD, Hubscher SG. Macsween's pathology of the liver $7^{\text {th }}$ ed 2018 .

82. Heymann F, Peusquens J, Ludwig-Portugall I, et al. Liver inflammation abrogates immunological tolerance induced by kupffer cells. Hepatology. 2015;62(1):279-291

83. Morrison MC, Kleeman R. Role of macrophage migration inhibitory factor in obesity, insulin resistance, type 2 diabetes, and associated hepatic comorbities: a comprehensive review of human and rodent studies. Front Immunol. 2015;6:308.

84. Morgantini C, Jaget J, Li X, et al. Liver macrophages regulate systemic metabolism through non inflammatory factors. Nat Metab. 2019;1(4):445459 .
85. Alzaid F, Lagadee F, Albuquerque M, et al. IRF5 governs liver macrophage activation that promotes hepatic fibrosis in mice and human. JCI Insight. 2016;1(20):e88689.

86. Xiao C, Stahel P, Mortgantini P, et al. Glucagon-like peptide-2 mobilizes lipids from the intestine in a systemic nitric oxide -independent mechanisms. Diabetes Obes Metab. 2019;21(11):2535-2541.

87. Rausch ME, Weisberg S, Vardhana P, et al. Obesity in C57BL/6J mice is characterized by adipose tissue hypoxia and cytotoxic T-cell infiltration. Int J Obes. 2008;32(3):451-463.

88. Murano I, Barbatelli G, Parisani V, et al. Dead adipocytes, detected as crown like structures, are prevalent in visceral fat depots of genetically obese mice. J Lipid Res. 2008;49(7):1562-1568.

89. Boutens L, Stienstra R. Adipose tissue macrophage going off track during obesity. Diabetologia. 2016;59(5):879-894.

90. Shi H, Kokoeva MV, Inouye K, et al. TLR4 links innate immunity and fatty acid-induced insulin resistance. $J$ Clin Invest. 2006;116(11):30153025 .

91. Shin KC, Hwang I, Choe SS, et al. Macrophage VLDLR mediates obesity induced insulin resistance with adipose tissue inflammation. Nat Commun. 2017;8(1):1087.

92. Wen H, Ting JP, O'Neil LA. A role for the NLRP3 inflassome in metabolic diseases and did warburg miss inflammation? Nat Immunol. 2012;13(4):352-357.

93. Wen H, Gris D, Lei Y, et al. Fatty acid-induced NLRP3ASC inflassome activation interferes with insulin signaling. Nat Immunol. 2011;12(5):408 415.

94. Hotamisligil GS, Peraldi P, Budavari A, et al. IRS-1-mediated inhibition of insulin receptor tyrosine kinase activity in TNF- $\alpha$ and obesity induced insulin resistance. Science. 1996;271(5249):665-668.

95. Haeusler RA, McGraw TE, Accili D. Biochemical and cellular properties of insulin receptor signaling. Nat Rev Mol Cell Biol. 2018;19(1):31-44

96. Kanety H, Feinstein R, Papa MZ, et al. Tumor necrosis factor alpha induced phosphorylation of insulin receptor substrate 1(IRS-1 ).possible mechanism for suppression of insulin stimulated tyrosine phosphorylation of IRS-1. J Biol Chem. 1995;270(40):23780-23784.

97. Jager J, Gremeaux T, Cormont $\mathrm{M}$, et al. Interleukin 1-beta induced insulin resistance in adipocyte through downregulation of insulin receptor substrate 1 expression. Endocrinology. 2007;148(1):241-251.

98. Wunderlich CM, Hovelmeyer N, Wunderlich FT. Mechanisms of chronic JAK-STAT-SOCS3 signaling in obesity. JAK-STAT. 2013;2(2):e23878.

99. Munder M, Eichmann K, Modolell M. Alternative metabolic states in murine macrophages reflected by the nitric oxide synthase/arginase balance: competitive regulation by CD 4+T cells correlates with Th1/Th2 phenotype. J Immunol. 1998;160(11):5347-5354.

100. Oren R, Farnham AE, Saito K, et al. Metabolic patterns of three types of phagocytosing cells. J Cell Biol. 1963;17(3):487-501.

101. Pavlou S, Wang L, Xu H, et al. Higher phagocytic activity of thioglycollateelicited peritoneal macrophages is related to metabolic status of the cells. J Inflamm (Lond). 2017;14:4.

102. Kellett DN. 2Deoxyglucose and inflammation. J Pharm Pharmacol. 1966;18(3):199-200.

103. Freemerman AJ, Johnson AR, Sacks GN, et al. Metabolic reprogramming of macrophages glucose transporter 1(GLUT 1)-mediated glucose metabolism drives a proinflammatory phenotype. $J$ Biol Chem. 2014;289(11):7884-7896.

104. Blouin CC, Page EL, Soucy GM, et al. Hypoxic gene activation by lipopolysaccharide in macrophages: implication of hypoxia inducible factor 1 alpha. Blood. 2004;103(3):1124-1130. 
105. Rodriguez-Prados JC, Traves PG, Cuenca J, et al. Substrate fate in activated macrophages: a comparison between innate, classic, and alternative activation. J Immunol. 2010;185(1):605-614.

106. Hedl M, Yan J, Witt H, et al. IRF5 is required for bacterial clearance in human M1 polarized macrophages and IRF5 immune mediated disease risk variants modulate this outcome. J Immunol. 2019;202(3):920-930.

107. Palson-McDermott EM, Curtis AM, Goel G, et al. Pyruvate kinase M2 regulates Hif- $1 \boldsymbol{\alpha}$ activity and IL-1 $\boldsymbol{\beta}$ induction and is a critical determinant of the warburg effect in LPS-activated macrophages. Cell Metab. 2015;21(1):65-80.

108. Luo W, Hu H, Chang R, et al. Pyruvate kinase M2 is a PHD3-stimulated coactivator for hypoxia-inducible factor 1. Cell. 2011;145(5):732-744.

109. Wolf AJ, Reyes CN, Liang W, et al. Hexokinase is an innate immune receptor for the detection of bacterial peptidoglycan. Cell. 2016;166(3):624-636.

110. Ip WKE, Hoshi N, Shouval DS, et al. Anti-inflammatory effect of IL10 mediated by metabolic reprogramming of macrophages. Science. 2017;356(6337):513-519.

111. Baardman I, Verberk SGS, Prange KHM, et al. A defective pentose phosphate pathway reduces inflammatory macrophages response during hypercholesterolemia. Cell Rep. 2018;25(8):2044-2052.

112. Infantino V, Convertini P, Cucci L, et al. The mitochondrial citrate carrier: a new player of inflammation. Biochem J. 2011;438(3):433-436.

113. Infantino V, Iacobazzi V, Palmieri F, et al. ATP citrate lyase is essentia for macrophage inflammatory response. Biochem Biophys Res Commun. 2013;440(1):105-111.

114. Wei X, Song H, Yin L, et al. Fatty acid-synthesis configures the plasma membrane for inflammation in diabetes. Nature. 2016;539(7628):294 298.

115. Hooftman A, O'Neil LA. The immunomodulatory potential of the metabolite itaconate. Trends Immunol. 2019;40(8):687-698.

116. Quinlan CL, Orr AL, Perevoschikova IV, et al. Mitochondrial complex II can generate reactive oxygen species at high rates in both the forward and reverse reactions. $J$ Biol Chem. 2012;287(32):27255-27264.

117. Tannahill G, Curtis A, Adamik J, et al. Succinate is an inflammatory signa that induces IL-1 $\boldsymbol{\beta}$ through HIF-1 $\alpha$. Nature. 2013;496((7444):238-242.

118. Langston PK, Nambu A, Jung J, et al. Glycerol phosphate shuttle enzyme GPD2 regulates macrophage inflammatory responses. Nat Immunol. 2019;20(9):1186-1195

119. Liu PS, Wang H, Li X, et al. $\alpha$-ketoglutarate orchestrates macrophage activation through metabolic and epigenetic reprogramming. Nat Immunol. 2017;18(9):985-994.

120. Vats D, Mukundan L, Odegaard JI, et al. Oxidative metabolism and PGC-1 beta attenuates macrophage mediated inflammation. Cell Metab. 2006;4(1):13-24.

121. Wang F, Zhang S, Vuckovic I, et al. Glycolytic stimulation is no a requirement for M2 macrophage differentiation. Cell Metab. 2018;28(3):463-475

122. Covarrubias AJ, Aksoylar HI, Yu J, et al. Akt-mTORC1 signaling regulates acly to integrate metabolic input to control of macrophage activation. Elife. 2016;5:e11612

123. Haschemi A, Kosma P, Gille L, et al. The sedoheptulose kinase CARKL directs macrophage polarization through control of glucose metabolism. Cell Metab. 2012;15(6):813-826.

124. Tavakoli S, Downs K, Short JD, et al. Characterization of macrophage polarization states using combined measurement of 2-Deoxyglucose and glutamine accumulation: implications for imaging of atherosclerosis. Arterioscler Thromb Vasc Biol. 2017;37(10):1840-1848.
125. Jha AK, Huang SC, Sergushichev A, et al. Network integration of parallel metabolic and transcriptional data reveals metabolic modules that regulate macrophage polarization. Immunity. 2015;42(3):419-430.

126. Schulman JG. Liver X receptors link lipid metabolism and inflammation. FEBS Lett. 2017;591(19):2978-2991.

127. Oishi Y, Spann NJ, Link VM, et al. SREBP1 contributes to resolution of proinflammatory TLR4 signaling by reprogramming fatty acid metabolism. Cell Metab. 2017;25(2):412-427.

128. Boutens L, Hooiveld GJ, Dhingra S, et al. Unique metabolic activators of adipose tissue macrophage in obesity promotes inflammatory responses. Diabetologia. 2018;61(4):942-953.

129. Ren W, Xia Y, Chen S, et al. Glutamine metabolism in macrophages; a novel target for obesity/ type2 diabetes. Adv Nutr. 2019;10(2):321-330.

130. Brennan JJ, Gilmore TD. Evolutionary origins of toll like receptors signaling. Mol Biol Evol. 2018;35(7):1576-1587.

131. Ermis-Karaali Z, Candan G, Aktuglu MB, et al. Toll like receptors 2 (TLR2) gene polymorphisms in type2 diabetes. Cell J. 2019;20(4):559-563.

132. Haversen L, Danielsson KN, Fogelstrand L, et al. Induction of proinflammatory cytokines by long chain saturated fatty acids in human macrophages. Atherosclerosis. 2009;202(2):382-393.

133. Zhao GN, Jiang DS, Li H. Interferon regulatory factors at the crossroads of immunity, metabolism, and disease. Biochym Biophys Acta. 2015;1852(2):365-378.

134. Chen W, Royer WE Jr. Structural insights into interferon regulatory factor activation. Cell Signal. 2010;22(6):883-887.

135. Orr JS, Puglisi MJ, Ellacott KL, et al. Toll-like receptors 4 deficiency promotes the alternatively activation of adipose tissue macrophages. Diabetes. 2012;61(11):2718-2727.

136. Kumari M, Wang X, Lantier L, et al. IRF3 promotes adipose inflammation and insulin resistance and repress browning. J Clin Invest. 2016;126(8):2839-2854.

137. Gunthner R, Anders HJ. Interferon regulatory factors determine macrophages phenotype polarization. Mediators Inflamm. 2013;2013:731023.

138. Eguchi J, Kong X, Tenta M, et al. Interferon regulatory factor 4 regulates obesity-induced inflammation through regulation of adipose tissue macrophages polarization. Diabetes. 2013;62(10):3394-3403.

139. Ameyar M, Wiszniewska M, Weitzman JB. A role for AP-1 in apoptosis: the case for and against. Biochimie. 2003;85(8):747-752.

140. Vesely PW, Staber PB, Hoefflers G, et al. Translational regulation mechanisms of AP-1 proteins. Mutat Res. 2009;682(1):7-12.

141. Takahashi M, Yagu H, Tazoe F, et al. Macrophage lipoprotein lipase modulates the development of atherosclerosis but not adiposity. J Lipid Res. 2013;54(4):1124-1134

142. Tuncman G, Hirosumi J, Solinas G, et al. Functional in vivo interactions between JNK1 and JNK2 isoforms in obesity and insulin resistance. Proc Natl Acad Sci USA. 2006;103(28):10741-10746.

143. Solinas G, Vilcu C, Neela JG, et al. JNK1 in haematopoietically derived cells contributes to diet induced inflammation and insulin resistance without affecting obesity. Cell Metab. 2007;6(5):386-397.

144. Solinas G, Becattini B. JNK at the crossroads of obesity, insulin resistance and cell stress response. Mol Metabol. 2017;6(2):174-184.

145. Xanthopoulea S, Curfs DM, Hofker HM, et al. Nuclear kappa B signaling in macrophages function and atherogenesis. Curr Opin Lipidol. 2005;16(5):536-542.

146. Arkan MC, Hevenr AL, Greten FR, et al. IKK beta links Inflammation to obesity induced insulin resistance. Nat Med. 2005;11(2):191-198. 
147. Wang N, Liang HW, Zen K. Molecular mechanisms that influence the macrophage m1-m2 polarization balance. Front Immunol. 2014;5:614

148. Yin Z, Ma TT, Lin Y, et al. IL-6/STAT3 pathway mediates M1/M2 macrophage polarization during the development of hepatocellular carcinoma. J Cell Biochem. 2018;119(11):9419-9432.

149. Coats BR, Schoenfelt KQ, Barbosa-Lorenza VC, et al. Metabolically activated adipose tissue macrophages perform detrimental and beneficial functions during diet induced obesity. Cell Rep. 2017;20(13):3149-3161.

150. Reardon CA, Lingaraju A, Schoenfelt KQ, et al. Obesity and insulin resistance promote atherosclerosis through an IFN $\gamma$ - regulated macrophage protein network. Cell Rep. 2018;23(10):3021-3030.

151. Vasametti SB, Karnewar S, Kanugula AK, et al. Metformin inhibits monocyte to macrophage differentiation via AMPK mediated inhibition of STAT3 activation: potential role in atherosclerosis. Diabetes. 2015;64(6):2028-2041

152. Tang C, Houston BA, Sorey C, et al. Both STAT 3 activation and cholesterol efflux contribute to the anti-inflammatory effects of apoA1ABCA1 interaction in macrophages. J Lipid Res. 2016;57(5):848-857.

153. Lee WJ, Tateya S, Cheng AM, et al. M2 macrophage polarization mediates anti-inflammatory effects of endothelial nitric oxide signaling. Diabetes. 2015;64(8):2836-2846.

154. Ricardo-Gonzalez RR, Red Eagle A, Odegaard JL, et al. IL-4/ STAT6 immune axis regulates peripheral nutrient metabolism and insulin sensitivity. Proc Natl Acad Sci USA. 2010;107(52):22617-22622.

155. Shu H, Wong B, Zhou G, et al. Activation of PPARalpha or gamma reduces secretion of matrix metalloproteinase 9 but not Interleukin 8 from human monocytic THP-1 cells. Biochym Biophys Res Commun. 2000; 267(1):345-349.

156. Ye G, Gao H, Wang Z, et al. PPAR $\alpha$ and PPAR $\gamma$ activation attenuates total free fatty acid and triglyceride accumulation in macrophages via the inhibition of Fatp1 expression. Cell Death Dis. 2019;10(2):39.

157. Risérus U, Sprecher D, Johnson T, et al. Activation of peroxisome proliferator-activated receptor (PPAR) delta promotes reversal of multiple metabolic abnormalities, reduces oxidative stress, and increases fatty acid oxidation to moderately obese men. Diabetes. 2008;57(2):332-339.

158. Kang K, Reilly SM, Karabacak V, et al. Adipocyte-derived Th2 cytokines and myeloid PPARdelta regulate macrophage polarization and insulin sensitivity. Cell Metab. 2008;7(6):485-495.

159. Lefterova ML, Steger DL, Zhou D, et al. Cell specific determinants of peroxisome proliferator-activated receptor gamma function in adipocytes and macrophages. Mol Cell Biol. 2010;30(9):2078-2089.

160. Jiang C, Ting AT, Seed B. PPAR-gamma agonists inhibit production of monocyte inflammatory cytokines. Nature. 1998;391(6662):82-86.

161. Wang GL, Jiang BH, Rue EA, et al. Hypoxia-inducible factor 1 is a basichelix-loop-helix-PAS heterodimer regulated by cellular $\mathrm{O}_{2}$ tension. Proc Natl Acad Sci USA. 1995;92(12):5510-5514.

162. Lewis JS, Lee JA, Underwood JC, et al. Macrophage responses to hypoxia: relevance of disease mechanisms. J Leukoc Biol. 1999;66(6):889-900.

163. O’Neil LA, Pearce EJ. Immunometabolism governs dendritic cells and macrophage function. $J$ Exp Med. 2016;213(1):15-23.

164. Odegaard JL, Ricardo-Gonzalez RR, Goforth MH, et al. Macrophagespecific PPARgamma controls alternative activation and improves insulin resistance. Nature. 2007;447(7148):1116-1120.

165. Chinetti G, Fruchart JC, Staels B. Peroxisome proliferator-activated receptor (PPARs): nuclear receptors with functions in the vascular wall. $Z$ Kardiol. 2001;90 Suppl 3:125-132.

166. Sugil S, Olson P, Sears DD, et al. PPARgamma activation in adipocytes is sufficient for systemic insulin sensitization. Proc Natl Acad Sci USA. 2009; 106(52):22504-22509.
167. Ricote M, Glass CK. PPARs and molecular mechanisms of transrepression. Biochym Biophys Acta. 2007;1771(8):926-935.

168. Kiss M, Czimmerer Z, Nagy L. The role of lipid-activated nuclear receptors in shaping macrophages and denditic cell function: from physiology to pathology. J Allergy Clin Immunol. 2013;132(2):264-286.

169. Repa JJ, Berge KE, Pomajzl C, et al. Regulation of ATP binding cassette sterol transporters ABCG5 and ABCG8 by the liver X receptors alpha and beta. J Biol Chem. 2002;277(21):18793-18800.

170. Fuentes L, Roszer T, Ricote M. Inflammatory mediators and insulin resistance in obesity: role of nuclear receptors signaling in macrophages. Mediators Inflamm. 2010;2010:319583.

171. Ghidetti S, Huang W, Ogawa S, et al. Parallel SUMOylation-dependent pathways mediate gene- and signal-specific transrepression by LXRs and PPARgamma. Mol Cell. 2007;25(1):57-70.

172. Li P, Spann NJ, Kaikkonen MU, et al. NCoR repression of LXR's restricts macrophage biosynthesis of insulin-sensitizing omega 3 fatty acids. Cell. 2013;155(1):200-214

173. Takikawa A, Mahmood A, Nawaz A, et al. HIF1 $\alpha$ in myeloid cells promotes adipose tissue remodeling towards insulin resistance. Diabetes. 2016;65(12):3649-3659.

174. Glass CK, Saijo K. Nuclear receptors transrepression pathways that regulate inflammation in macrophages and $\mathrm{T}$ cells. Nat Rev Immunol. 2010;10(5):365-376.

175. Treuter E, Venteclef N. Transcriptional control of metabolic and inflammatory pathways by nuclear receptors SUMOylation. Biochym Biophys Acta. 2011;1812(8):909-918.

176. Chen X, Barozzi I, Termaninin A, et al. Requirement for the histone deacetylase Hdac3 for the inflammatory gene expression program in macrophages. Proc Natl Acad Sci USA. 2012;109(42):2865-2874.

177. Fan R, Toubal A, Goni S, et al. Loss of the corepressor GPS2 sensitizes macrophage activation upon metabolic stress induced by obesity and type2 diabetes. Nat Med. 2016;22(7):780-791.

178. Drareni K, Ballaire R, Barilla S, et al. GPS2 deficiency triggers maladaptive white adipose tissue expansion in obesity via HIF1A activation. Cell Rep. 2018;24(11):2957-2971.

179. Liang N, Damadipoulous A, Goni S, et al. Hepatocyte-specific loss of GPS2 in mice reduces non-alcoholic steatohepapititis via activation of PPAR alpha. Nat Commun. 2019;10(1):1684.

180. Coppo M, Chinenov Y, Sacta MA, et al. The transcriptional coregulator GR1P1 controls macrophage polarization and metabolic homeostasis. Nat Commun. 2016;7:12254.

181. Zhang X, Wang Y, Yuan J, et al. Macrophage/microglial Ezh2 facilitates autoimmune inflammation through inhibition of Socs3. J Exp Med. 2018;215(5):1365-1382.

182. Satoh T, Takeuchi O, Vandenbon A, et al. The Jmjd3-Irf4 axis regulates M2 macrophage polarization and host responses against helminth infection. Nat Immunol. 2019;11(10):936-944.

183. De Santa F, Narang V, Yap ZH, et al. Jmjd3 contributes to the control of gene expression in LPS activated macrophages. EMBO J. 2009;28(21):3341-3352.

184. Gallagher KA, Joshi A, Carson WF, et al. Epigenetic changes in bone marrow progenitor cells influence the inflammatory phenotype and alter wound healing in type 2 diabetes. Diabetes. 2015;64(4):1420-1430.

185. Niziachristos P, Tsirigos A, Welstead GG, et al. Contrasting roles of histone 3 lysine 27demethylases in acute lymphoblastic leukaemia. Nature. 2014;514(7523):513-517.

186. Wahl S, Drong A, Lehne B, et al. Epigenome wide associations study of body mass index, and the adverse outcomes of adiposity. Nature. 2017;541(7635):81-86 
187. Pollack RM, Donath MY, Le Roith D, et al. Anti inflammatory agents in the treatment of diabetes and its vascular complications. Diabetes Care. 2016;39(Suppl 2):S244-S252.

188. Rekedal LR, Massarotti E, Garg R, et al. Changes in glycosylated haemoglobin after initiation of hydroxyl chloroquine or methotrexate treatment in diabetes patients with rheumatic diseases. Arthritis Rheum. 2010;62(12):3569-3573.
189. Orliaguet L, Dalmas E, Drareni K, et al. Mechanisms of macrophage polarization in insulin signaling and sensitivity. Front Endocrinol (Lausanne). 2020;11:62.

190. Russo L, Lumeng CN. Properties and functions of adipose tissue macrophages. Immunology. 2018;155(4):407-417. 